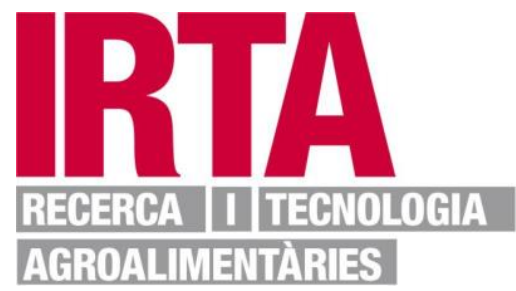

This document is a postprint version of an article published in Journal of Proteomics $\subset$ Elsevier after peer review. To access the final edited and published work see https://doi.org/10.1016/j.jprot.2020.103677

Document downloaded from: 


\section{Metabolome and Proteome Changes in Skeletal Muscle and Blood of Pre-weaning Calves fed Leucine and Threonine Supplemented Diets}

Kuai $\mathrm{Yu}^{1}$, Manolis Matzapetakis ${ }^{2}$, Anita Horvatić ${ }^{3}$, Marta Terré ${ }^{4}$, Alex Bach ${ }^{4,5}$, Josipa Kuleš

${ }^{3}$, Natalia Yeste ${ }^{1}$, Néstor Gómez ${ }^{1}$, Laura Arroyo ${ }^{1}$, Elisabet Rodríguez-Tomàs ${ }^{6}$, Raquel Peña ${ }^{7}$, Nicolas Guillemin ${ }^{3}$, André M. de Almeida ${ }^{8}$, Peter David Eckers ${ }^{\text {11 }}$ - 9 , Anna Bassols ${ }^{1,7}$

1 Departament de Bioquímica i Biologia Molecular, Fa`uı tt de Veterinària, Universitat Autònoma de Barcelona (Cerdanyola del Vallès, 0`'9', Barcelona), Spain.

2 ITQB NOVA, Instituto de Tecnologia Quín: ca e Biológica António Xavier, Universidade Nova de Lisboa, Oeiras, Portugal.

3 ERA Chair team, Faculty of $V$ ternary Medicine, University of Zagreb (Heinzelova 55, 10000, Zagreb), Croatia.

4 Departament of Rumina at Production, Institut de Recerca i Tecnologia Agroalimentàries Caldes de Montbui, 08140, Barcelona, Spain.

5 Catalan Institution for Research and Advanced Studies (ICREA), Barcelona, Spain.

6 Biomedical Research Center, University Hospital S. Joan, Reus, Spain.

7 Servei de Bioquímica Clínica Veterinària, Facultat de Veterinària, Universitat Autònoma de Barcelona Cerdanyola del Vallès, 08193, Barcelona, Spain. 
8 LEAF, Instituto Superior de Agronomia, Universidade de Lisboa, Lisbon, Portugal.

9 Institute of Biodiversity, Animal Health and Comparative Medicine, University of Glasgow, Glasgow, UK.

Corresponding author: anna.bassols@uab.cat 


\begin{abstract}
In pre-weaning calves, both leucine and threonine play important roles in growth and muscle metabolism. In this study, metabolomics, proteomics and clinical chemistry were used to assess the effects of leucine and threonine supplementation added to milk replacer on 14 newborn Holstein male calves: 7 were fed a control diet (Ctrl) and 7 were fed the Ctrl diet supplemented with $0.3 \%$ leucine and $0.3 \%$ threonine (LT) from 5.6 days of age $t$, 53.6 days. At this time, blood and semitendinosus muscle biopsies were collected for an lys 1 . Integrated metabolomics and proteomics showed that branched-chain amino acids ( $\mathrm{PC} \wedge \mathrm{A}$ ) degradation and mitochondrial oxidative metabolism (citrate cycle and respiratory cha $a^{i}$ ) $\mathrm{w}$ se the main activated pathways in muscle because of the supplementation. BCAA de iv ، tives and metabolites related to lipid mobilization showed the major changes. Tr a ilt arious effects of activated oxidative phosphorylation were balanced by the יpreguı tion of antioxidant proteins. An increase in protein synthesis was indicated by elc va “ ${ }^{\mathrm{N}}$ aminoacyl-tRNA biosynthesis and increased S6 ribosomal protein phosphorylatirn $\eta$ skeletal muscle. In conclusion, LT group showed greater BCAA availability and mito hor 'rial oxidative activity; as the muscle cells undergo greater aerobic metabolism, a ${ }^{\prime}+i c$ id $: n t$ defenses were activated to compensate for possible cell damage. Data are available via FıvıeomeXchange (PXD016098).
\end{abstract}

\title{
Keywords
}

Amino acid; calf; muscle; plasma; metabolomics; proteomics 


\section{Introduction}

Amino acids (AA) are important for the growth of pre-weaning calves being pivotal for tissue development, endocrine, and immune function [1,2]. Leucine is an essential AA and one of the more studied branched-chain AA (BCAA) in several species due to its function in promoting protein synthesis through the mammalian target of rapamycin (mTOR) pathway in skeletal muscle [3,4]. Leucine also enhances energy homeostasis through i $\urcorner$ rreasing fatty acid oxidation and provides skeletal muscles with an increased flux of lipids to supp ly energy and intermediary substrates to support protein synthesis [5]. Leucine from di $t_{i}{ }^{*} v r$ rotein can bypass metabolism in the liver, which results in a rise of plasma leucine le $\mathrm{e}^{\mathrm{r}}$ ls a $\mathrm{nd}$ the activation of leucine signaling in peripheral tissues in response to a meal [6] and, al ong these tissues/organs, the skeletal

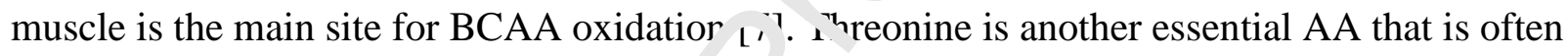
considered limiting in milk replacers (MR) for zalves [8]. It participates in the synthesis of several other important AA such as $\mathrm{g}^{\prime} \mathrm{y}_{1} \mathrm{1}^{\circ}$ and serine which are involved in the production of

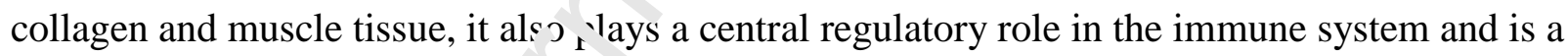
major component of intestin?l m...ins and plasma $\gamma$-globulins [1,9].

To understand the role of utritional compounds at the molecular level [10] and to analyze the coordination of multiple systems including for instance the immune, digestive and endocrine, the identification of large sets of biological molecules is required. Accordingly, "multi-omics" approaches have gained popularity in animal science. The combination of proteomics and metabolomics in food and nutrition science, more recently named as "foodomics", has been shown to be a more suitable approach compared to other "-omics" such as genomics or transcriptomics [10]. 
The objective of this work is to use a foodomics-based approach in calves' skeletal muscle and plasma in order to fully assess the effects of leucine and threonine supplementation in the milk replacer (MR) during the pre-weaning period, under the hypothesis that AA supplementation will modify the energy metabolism, especially in the muscle tissue. Furthermore, the activation of the mTOR pathway was studied in skeletal muscle by determining the phosphorylation of S6 ribosomal protein, a key event in protein synthesis.

\section{Materials and Methods}

\section{Animal Housing and Diet Management}

Animal management followed the recomr end tions of the Animal Care Committee of Institut de Recerca i Tecnologia Agroalimentàries , 'TRA) under the approval research protocol FUE-201700587321, authorization code 9733

Fourteen Holstein male calves $(11.9 \pm 0.67 \mathrm{~kg}$ of body weight (BW) at age of $5.6 \pm 0.67$ days) were obtained from Gran a N'urucuc (Gurb, Barcelona, Spain) and housed individually at IRTA Torre Marimon (Caldes d'Montbui, Barcelona, Spain) and fed a milk protein-based MR twice a day. The MR contained 24\% crude protein (based in skimmed milk protein and whey protein concentrate) and $20.3 \%$ fat, on a DM basis. Calves were fed 4 L/day at $15 \%$ dry matter (DM) concentration from days 1 to 7 of experiment. Then, MR volume was increased to $6 \mathrm{~L} / \mathrm{day}$ from 8 to 49 days of age, and it was reduced to one single feeding of $3 \mathrm{~L} /$ day until animals were weaned. Calves were divided into control $(\mathrm{Ctrl}, \mathrm{n}=7)$ and $\mathrm{Ctrl}$ supplemented with $0.3 \%$ leucine and $0.3 \%$ threonine $(\mathrm{LT}, \mathrm{n}=7)$ groups according to the AA profile in the MR formula. 
Ingredient and chemical composition are shown in Supplementary material (Table S 1). All AA were provided by Livzon Group (Fuxing Pharmaceutical, Ningxia, China). All animals had free access to water, and restricted access to a pelleted concentrate starter feed to minimize the impact of concentrate feeding on AA supply. The starter feeding program is shown in Supplementary material (Table S 2). Chopped barley straw was offered ad libitum during the study.

\section{Sample Collection}

At $53.6 \pm 0.67$ days of age and $81.8 \pm 3.49 \mathrm{~kg}$ of BW, blood was $\mathrm{ht}$, ined from the jugular vein, $4 \mathrm{~h}$ after the morning MR feeding. Plasma and serum were bbtained by centrifugation at $1,500 \mathrm{~g}$ for $10 \mathrm{~min}$. Plasma and serum were aliquoted and storea ot $80^{\circ} \mathrm{C}$ until further analysis. Biopsies were obtained from the semitendinosus r $v \cdot s e$ as previously described [11] and kept at $-80^{\circ} \mathrm{C}$ until further analysis. Prior to Omi s a says, frozen muscle tissues were ground into a fine powder with mortar and pestle in h.' 'id nitrogen and at least two $0.5 \mathrm{~g}$ aliquots were made and kept in a $15 \mathrm{ml}$ polypropylene vial it $80{ }^{\circ} \mathrm{C}$.

\section{Serum Clinical Chemistry an +1 'asma AA}

Serum clinical chemisı.y aniytes were determined with an Olympus AU400 analyzer (Olympus corp., Tokyo, Japan). Methods, reagents and intra-assay CV (\%) were previously reported [12]. Plasma AA were determined by HPLC as described [11] with some modifications. Briefly, an Elite LaChrom (Hitachi, Tokyo, Japan) equipped with an UV detector (Hitachi L-24200, Tokyo, Japan) with a Novapak C18 column (300 mm x $3.9 \mathrm{~mm}$ ) from Waters (Milford, MA, USA) were used. The flow rate was $1.0 \mathrm{ml} / \mathrm{min}$, and the column temperature was kept at $42{ }^{\circ} \mathrm{C}$. The injection volume was $10 \mu \mathrm{L}$ and the detection wavelength was set at $254 \mathrm{~nm}$. The solvent system consisted of two eluents: (A) $140 \mathrm{mM}$ sodium acetate trihydrate, $12 \mathrm{mM}$ TEA and $1 \mathrm{mg} / \mathrm{L}$ EDTA at $\mathrm{pH}$ 
5.02 and (B) 60\% acetonitrile/40\% water. The Agilent software EZChrom Elite system V3.1.7 (Santa Clara, CA, USA) was used for system control and data acquisition.

\section{Proteomic Analysis}

\section{Protein Extraction and Quantification}

One aliquot of semitendinosus muscle fine powder in a $15 \mathrm{~mL}$ tube was sonicated in $3 \mathrm{~mL}$ extraction buffer (137 mM NaCl, $50 \mathrm{mM}$ HEPES, 2\% SDS, 1\% $\stackrel{\mathrm{m}}{\mathrm{m}} 4 \mathrm{c}, 10 \%$ glycerol, $2 \mathrm{mM}$ $\mathrm{Na}_{3} \mathrm{VO}_{4}, 100 \mathrm{mM} \mathrm{NaF}$ and $1 \%$ protease inhibitor cocktail (S gma), pH 7.4) at $40 \%$ amplitude following a sequence of 10-s on and 5-s off, three times mi ic. The homogenate was centrifuged at $12,000 \mathrm{~g}$ for $15 \mathrm{~min}$ at $4^{\circ} \mathrm{C}$ and the supernatant (mus cle t. tract) retrieved. Protein concentration was determined using the $\operatorname{Bradfo}^{-1}$ mu mod. Samples were kept at $-80^{\circ} \mathrm{C}$ until analyses.

Protein Immunoblot for S6 and Phosp $h$, - iv in Muscle

All antibodies were purchased fru $\eta$ Cell Signaling Technology (Denvers, MA, USA). $15 \mu \mathrm{g}$ of muscle extract per samplf w.re subjected to $12 \%$ SDS-PAGE and transferred to PVDF membranes. Total protein taining (TSP) by Ponceau S was performed for band intensity normalization on membrane after transfer. The membranes were then immunoblotted with primary antibodies (1:2000) against S6 ribosomal protein (\#2217) and phospho-S6 ribosomal protein (\#4858). The dilution for secondary antibodies were 1:5000 and 1:2000 for S6 and phospho-S6, respectively. Immunoblot bands were processed using Multi Gauge software (Fujifilm, Tokyo, Japan). Each sample was normalized by dividing the band density by its normalization factor. The latter was in turn calculated taking into account the mean TPS density 
of all gels and the mean TPS density of the gel where the sample was placed. Immunoblots were repeated three times with a random distribution of samples between gels.

Sample Preparation for High Resolution LC-MS/MS

Muscle samples were processed using Filter-Aided Sample Preparation (FASP) with some modifications [13]. Briefly, for each sample and internal standard (pooled sample), $35 \mu \mathrm{g}$ of proteins were diluted in UA buffer ( $8 \mathrm{M}$ urea in $0.1 \mathrm{M}$ Tris/HCl, p. 8.5) to $200 \mu \mathrm{L}$. They were then added to a $10 \mathrm{kDa}$ filter unit (Microcon YM-10, Merck Milinn'e, Burlington, MA, USA), centrifuged at $13,000 \mathrm{~g}$ for $15 \mathrm{~min}$ at $20{ }^{\circ} \mathrm{C}$ and washed by 'daıg $200 \mu \mathrm{L}$ of UA buffer followed by centrifugation. Samples were alkylated with $100 \mu \mathrm{L} \wedge$ dr acetamide (0.05 M IAA in UA buffer) for $20 \mathrm{~min}$ in the dark at RT. Additional se $\mathrm{i}, \mathrm{s}$,f washes with $100 \mu \mathrm{L}$ of UA buffer (twice) and $100 \mu \mathrm{TEAB}(0.1 \mathrm{M}$ triethyla. $\mathrm{mr}$ niun bicarbonate) (twice) with subsequent centrifugation were conducted. Overnigi ${ }^{+}$digestion at $37{ }^{\circ} \mathrm{C}$ was performed with $50 \mu \mathrm{L}$ of $0.1 \mathrm{M}$ TEAB containing $2 \%$ trypsin (v/v). Try ot c peptides were eluted by centrifugation at $13,000 \mathrm{~g}$ for $10 \mathrm{~min}$ and washed with $50 ; \mathrm{L}$ o: buffer (50\% acetonitrile in $0.1 \mathrm{M} \mathrm{TEAB}, \mathrm{v} / \mathrm{v})$. Collected flowthrough was dried and us $>$ or TMT labelling. Before labelling, pellets were dissolved in 50 $\mu \mathrm{L}$ of $0.1 \mathrm{M}$ TEAB.

Plasma-EDTA samples were prepared as described [14] with some modifications. Briefly, $35 \mu \mathrm{g}$ of proteins per sample or internal standard were diluted with $100 \mu \mathrm{L} 0.1 \mathrm{M}$ TEAB containing $1 \%$ SDS, reduced, alkylated and acetone precipitated overnight. Protein pellets were collected by centrifugation and dissolved in $50 \mu \mathrm{L}$ TEAB containing $1 \mu \mathrm{g}$ trypsin for overnight digestion at $37^{\circ} \mathrm{C}$.

Tandem Mass Tag (TMT) Labelling for Muscle and Plasma Proteins 
TMTsixplex ${ }^{\mathrm{TM}}$ Isobaric Label Reagent Set (\#90061) from Thermo Fisher Scientific (Waltham, MA, USA) was used for peptide labelling. All reagents were prepared as previously reported $[14,15]$. Briefly, for the labelling, $19 \mu \mathrm{l}$ of reconstituted TMT reagent was added to each tryptic digested sample and incubated for $1 \mathrm{~h}$ at room temperature. Internal standard was labelled with the TMT $m / z 126$ while other tags were randomized. The reaction was quenched by adding $8 \mu 1$ of 5\% hydroxylamine to each sample and a 15 min incubation. For each sixplex, samples were combined at equal amounts, aliquoted, dried in a vacuum centrifuge ?nd kept in $-80^{\circ} \mathrm{C}$ until further analysis.

High Resolution LC-MS/MS Analyses and Bioinformati s

Mass spectrometric analysis was conducted on an 'J'ci) nate 3000 RSLCnano system (Dionex, Germering, Germany) and Q Exactive Plu . mז ss spectrometer (Thermo Fisher Scientific, Bremen, Germany) as reported [14]. In . hort, after desalting on the trap column, TMT-labelled peptides were separated on the analvtic $\mathrm{tl}$ :olumn (PepMap ${ }^{\mathrm{TM}}$ RSLC C18, $50 \mathrm{~cm} \times 75 \mu \mathrm{m}$ ) using a 120-min linear gradient of $5-5 \%$ mobile phase B $(0.1 \%$ formic acid in $80 \% \mathrm{ACN})$ at the flow rate of $300 \mathrm{~nL} / \mathrm{min}$. Peptides : rer : introduced into the mass spectrometer using a $10 \mu \mathrm{m}$-inner diameter SilicaTip emilır (ivew Objective, Woburn, MA, USA) in a nanospray Flex ion source. For DDA analysis, the Top-8 method was used.

Proteome Discoverer (version 2.0, Thermo Fisher Scientific, Waltham, MA, USA) was used for peptide identification and relative quantification. SEQUEST algorithm database search against Bos taurus FASTA files (NCBI database, 7/12/2017, 46105 entries) was performed. Proteins with at least two unique peptides and 5\% FDR were reported as validly identified. Furthermore, for reporter ion-based protein quantification, internal standards were used to compare the data 
between the TMT sixplexes. For bioinformatics, NCBI accession number (gi number) was

converted to gene symbol by Biological Database Network (https://biodbnet-

abcc.ncifcrf.gov/db/db2db.php) and protein function was explored with PANTHER

(http://pantherdb.org/).

\section{Validation}

PON1 esterase activity was measured in plasma-heparin samples $b$, analyzing the hydrolysis of phenyl acetate (arylesterase activity, ARE), as previously descrit - '16].

\section{Metabolomic Analysis}

\section{Muscle and Serum Extractions}

The extraction of the aqueous metabolites $\mathrm{L}^{\sim} \mathrm{n}$ muscle powder was performed as described [11].

For serum, $300 \mu \mathrm{l}$ of each sample were ex *acted by methanol protein precipitation method as

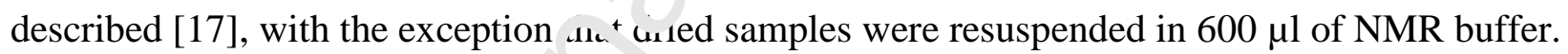

\section{Data Acquisition, Processin` an`'Analyses}

For muscle, data acquisil $>n$ method and parameters, data processing protocols and analyses were as described previously [11]. For serum samples, 128 scans and 8 test scans were applied. All raw data have been deposited to the EMBL-EBI MetaboLights database (https://www.ebi.ac.uk/metabolights/MTBLS1045).

\section{Spectral Profiling for Chemometrics}

All 1r data from either biological sample collected from Bruker were uploaded to NMRProcFlow 1.2 Online spectral processing tool interface [18]. Spectra were calibrated referring to the ppm 
and some peaks were aligned. $\mathrm{H}_{2} \mathrm{O}$ peak was zeroed. For bin data, peaks were bucketed following previous knowledge of assignment. Peak intensities for each sample were normalized by its own weight for muscle, no normalization for serum, and centered-scaled for both.

\section{Statistics}

All statistical analyses were performed using R (version 3.5.2) under RStudio (version 1.2.1335), "dplyr" [19], "reshape2" [20], "ggpubr" [21], "ggplot2" [22], "corr ${ }^{\prime}$ lot" [23], "nortest" [24] and "openxlsx" [25] packages were used for data manipulation, statis : ${ }^{c s}$ and graphic processes. Performance and intake data were analyzed using a mixed ffeus model accounting for the fixed effects of treatment, time of measurement, and their 2-w.vi ateraction, and the random effect of calf. Time entered the model as a repeated measur "ssi ig an autoregressive covariance matrix. Initial BW was used as a covariate in the $r$ od $f$. For statistical difference (univariate analyses) in the serum clinical chemistry, plasma $\mathrm{A}_{\mathrm{r}}$. and metabolomic results, a one-way analysis of variance (ANOVA) was used and for $w$ es ern blot and proteomic data, a Wilcoxon test was used, $p$ between 0.05 and 0.1 was ins ${ }^{n}{ }^{2}{ }^{\prime} t e{ }^{\prime}$, while $<0.05$ was considered significant. For MSproteomics, fold change betw:en two groups was calculated using the function of $\log 2\left(\right.$ Mean $(\mathrm{LT}) /$ Mean $\left(C^{+} \mathrm{r}_{1}\right)$ ). Absolute value of fold change $\geq 0.20$ and $p<0.05$ were considered significant. Additionally, some proteins/metabolites with $p$ between 0.05 and 0.1 or/and with fold changes between 0.10 and 0.20 showing significant correlations with representative metabolites, were also included in the analysis.

For univariate analyses in metabolomics, significant chemical shifts from chemometrics were inspected to assist the identification. For multivariate analyses, principle component analyses 
(PCA) with loadings of both quantified and chemometric data were plotted. The correlation plot in metabolomic results was made and Pearson $r$ was used as distance measurement.

All concentrations are presented in form of mean \pm SE.

\section{Proteomic-Metabolomic Interactive Analyses}

For significant proteins and metabolites, their official gene symbols (proteins) and compound names (metabolites) were analyzed using Reactome (version 69 ri: $7 s$ d on June 12, 2019) (https://reactome.org/) [26] and Joint Pathway Analysis prov` dea by MetaboAnalyst. Human (Homo sapiens) was selected as species since Bos tauru vis not available in both databases. The hypergeometric test, degree centrality and gene-m.tabc ite pathways options were used for enrichment, topology and pathway analyses, res.'eclively, in the MetaboAnalyst. For unannotated proteins, literature and Uniprot rere referred.

\section{Results}

\section{Performance results}

The mean average daily gain (ADG) was $0.89 \mathrm{~kg} / \mathrm{d}$ and $0.96 \mathrm{~kg} / \mathrm{d}$ in Ctrl and LT calves respectively. No significant differences between groups in ADG, BW or food intake were recorded. Complete results are shown in Supplementary material (Table S 3).

\section{Blood clinical chemistry and AA profile}


Analytes with significant differences between groups are shown in Table 1. Decreased GPx, increased cholesterol and a tendency for an increase in triglycerides were observed in the LT group. There was an increase in plasma isoleucine and histidine, and a tendency for an increase in leucine concentrations. Complete results are shown in Supplementary material (Table S 4). All blood biochemistry parameters were within the reference interval for this species [12].

\section{Proteomics in skeletal muscle and plasma}

The differentially abundant proteins in muscle and plasma from $\iota$ th groups are shown in Tables 2 and 3, respectively, and the raw proteomic data are presc tea in Supplementary material (Table S 5 for muscle and Table $\mathrm{S} 6$ for serum, respectively. Th $\mathrm{m}_{\mathrm{m}}$ ass spectrometry proteomics data have been deposited to the ProteomeXchange Con ir iti im via the PRIDE [27] partner repository with the dataset identifier PXD016098.

In muscle, nine proteins were found with . igher abundances (HADHA, PRDX1, SOD2, COX2, COX5, NDUFAB1, ACO2, SLC2 1 and VCP) and two with lower abundances (GYS1 and RTN2) in LT animals in companı on to Ctrl animals. For increased abundance proteins, GO analysis indicates a positi $\mathrm{e}$-gulation of ATP biosynthetic process (GO:2001171) and the removal of superoxide rac ${ }^{\circ}$ cals (GO:0019430) as the most relevant biological processes and the antioxidant activity (GO:0016209) as the first molecular function. Complete GO analysis is shown in Supplementary material (Table S 7).

In plasma, five proteins were found with higher abundance (APOA2, APOC3, CRP, F2 and PON1) in treated animals. The GO analysis for biological process showed that three of them (APOA2, APOC3 and PON1) are related to regulation of cholesterol transport (GO:0032374) and chylomicron assembly (APOA2 and APOC3) (GO:0034378). CRP and F2 are associated to 
the acute phase response (GO:0006953). The GO analysis for molecular function showed three proteins related to lipoprotein receptor binding (APOA2, APOC3 and PON1) (GO:0070325). Proteins with lower abundances in the LT group included six isoforms of the SERPINA3 protein (SERPINA3-1, -2, -5, -6, -7 and -8), ITIH4 and FN1. All eight proteins are involved in the GO peptidase regulator activity (GO:0061134) molecular function and all, with the exception of FN1 are also related to serine-type endopeptidase inhibitor activity (GO:0004867). Complete GO analysis is shown in Supplementary material (Table S 8).

For validation of proteomic results, plasma PON1 was deterr iner and found increased with a statistical tendency $(p=0.06)$ in LT $(125.55 \mathrm{U} / \mathrm{L} \pm 9.70$, cu npared to Ctrl (88.16 U/L \pm 11.58$)$.

\section{Phosphorylation status of S6 in skeletal muscle}

The ratio of Phospho-S6/S6 in muscle was s, nificantly increased in LT compared with Ctrl calves, as shown in Figure 1.

\section{Metabolomics in skeletal musc e a nd serum}

Quantified metabolites of ı1ı scie and serum with significant differences are shown in Tables 4 and 5 , respectively.

In muscle, two BCAA (leucine and valine), two citrate cycle-related metabolites (2-oxoglutarate and GTP) as well as NADH and acetoacetate were significantly increased in LT animals. Furthermore, acetate showed a tendency for increase.

In serum, seven proteinogenic AA (asparagine, histidine, isoleucine, leucine, proline, serine and valine) and one AA derivative (citrulline) were significantly increased in LT animals. Threonine 
showed a tendency to increase. Furthermore, catalytic metabolites derived from leucine (2oxoisocaproate), threonine (2-oxobutyrate) and valine (3-hydroxyisobutyrate) as well as carnitine and 3-hydroxybutyrate, two metabolites related to lipid degradation, were also elevated.

The PCA for the quantified metabolites with their corresponding loadings are shown in Figure 2 for muscle (a) and serum (b), respectively. Verification of consistency with the original data was performed by matching PCA of chemometrics from both biologicrl samples (Supplementary material Fig. S 1).

Correlation plots for all metabolites and proteins in musclc ana serum are shown in Figure 3a and $3 b$, respectively $(p<0.05)$. In muscle, leucine show $d s$ gnificant correlation with acetoacetate, carnitine as well as the other BCAA i $r$ l ucine and valine). Also a positive correlation appeared between the proteins ela' $2 d$ to mitochondria and OXPHOS (COX2, COX5A, VCP and NDUFAB1), and be veen leucine and HADHA. In serum, the correlation plot revealed several positive correlatic is between leucine and/or threonine and several AA. SerpinA3.1, SerpinA3.7, PON1 CK? and APOC3 also clustered together showing a positive correlation, as well as Serpin. 3. SerpinA3.6, SerpinA3.8, ITIH4 and APOA2. These two groups of proteins corre 'ttu negatively one to the other.

The analysis of the correlation between metabolites in serum and muscle (Supplementary material Fig. S 2) showed that muscular leucine and valine positively correlated with muscular and serum carnitine, and with serum citrulline and amino acids as isoleucine and lysine. Muscular NADP also correlated with several serum AA (isoleucine, leucine, lysine, proline, tryptophan, valine, phenylalanine). Negative correlations were observed between serum acetate and muscular glucose, glucose-1P and pyruvate; 2-oxobutyrate showed negative correlations 
with glucose, glucose-1P, glucose-6P and lactate; and serum lactate negatively correlated with muscular acetate.

\section{Pathway analysis}

The Reactome pathway for protein analysis yielded several significant pathways, in accordance with the GO ontology. The Reactome analysis for small molecul $\sigma_{s}$ (KLGG nomenclature) also presented coherent results (Supplementary material Table S ؟).

In muscle, citrate cycle and respiratory electron transp ${ }^{*} \cdot \mathrm{m}$.tochondrial fatty acid beta-oxidation and detoxification of reactive oxygen species wer ir entified with proteomic data. Among the most relevant for metabolites, BCAA catar Jlis $\mathrm{n}$ (?.-HSA-70895), metabolism of amino acids and derivatives (R-HSA-71291), synth sis of ketone bodies (R-HSA-77111), tRNA modification in the nucleus and cytosol (R-HSA-6, 8'3 i5) and citrate cycle (R-HSA-71403) were observed.

In plasma/serum, chylomicror as ` ${ }^{2}$ mbly and remodeling (R-HSA-8963888 and R-HSA8963901) and lipoprotein as nıly and remodeling (R-HSA-8963898 and R-HSA-8963899) were identified with prote mic data and tRNA aminoacylation (R-HSA-379724) and metabolism of AA and derivatives (R-HSA-71291) with small compounds.

The joint pathway analysis of increased abundance proteins and metabolites in blood and muscle developed with MetaboAnalyst are shown in Table 6. Four pathways (valine, leucine and isoleucine degradation; butanoate metabolism, aminoacyl-tRNA metabolism; and citrate cycle) were found with significant change, furthermore coherent with the Reactome analysis. 


\section{Discussion}

Schematics of the putative metabolic effects caused by leucine/threonine supplementation is shown in Figure 4. Detailed explanation is given below.

\section{Supplementation with leucine and threonine increases the conrentration of BCAA and their derivative metabolites}

Metabolomic analysis indicated increases in the three BC $\&$ $\uparrow$ in serum and muscle, which implies that leucine reached the blood and the muscle, and at a metabolic connection among BCAA exists. On the other hand, no increase in th $\bullet$ sn ne neither in blood nor muscle was observed, despite being supplemented at $\mathrm{t}^{\mathrm{t}} \mathrm{e}$ s $r$ me level as leucine. Serine was greatly increased, thus this may be explained by the rapid ` nnversion of threonine to serine.

Most AA are first metabolized in ${ }^{1}$ e $\mathrm{L}^{\mathrm{v}} \mathrm{v}$ er and turned into ketoacids by transamination. In contrast, BCAA are not initialı, ma tabolized in the liver, due to low hepatic activity of branchedchain-amino-acid aminot ansierase (BCAT). Thus, BCAA increase rapidly in the plasma after protein intake and are reac ily available to extrahepatic tissues [28]. Most of the BCAA are initially catabolized in the skeletal muscle, due to its high BCAT activity, which can reversibly transfer the alpha-amino group in BCAA to 2-oxoglutarate $(\alpha-\mathrm{KG})$ to form glutamate and the corresponding branched-chain keto acids (BCKA): 2-oxo-isocaproate (ketoleucine), 2-oxo-3methylvalerate (ketoisoleucine) and 2-oxo-isovalerate (ketovaline). Glutamate can further form alanine or glutamine to be released in the bloodstream whereas BCKA are catabolized mainly in the liver [29]. Since the three BCAA have parallel pathways for degradation, the finding of 
higher concentrations of 2-oxoisocaproate and 3-hydroxyisobutyrate in serum in the LT group indicates that these AA are indeed catabolized [30]. These AA and their metabolites ( $\alpha$ ketoisocaproic acid and $\beta$-hydroxy- $\beta$-methylbutyrate) have been reported to play important roles in enhancing muscle growth during the neonatal period [31]. Positive correlation between muscular leucine, isoleucine and valine with serum citrulline and aspartate, both intermediates of the urea cycle, may indicate a higher degradation of amino acids as a general event of the organism, in the last stage of metabolism of the amino group in the $\iota$ "ea cycle taking place in the liver.

\section{Supplementation with leucine and threonine affects 'tpr' metabolism}

Results from the proteomic analysis indicated an i ire se in plasma apolipoproteins APOA2 and APOC3, which are involved in lipid and $c^{\prime}$.ole teroi transport (GO:2000910 and GO:0060621) in the LT group. Furthermore, PON1, an e ryme that is involved in detoxification, is bound to high-density lipoproteins (HDL) in nla: $m_{\perp}$ [32]. The increase in plasma apolipoproteins is consistent with the increase in $T$. Is a.d cholesterol observed after conventional clinical chemistry assays. Regarding the ncrease in serum 3-hydroxybutyrate, it could likely be consequence of an incre: st in the production of butyrate in the rumen, since these young animals have already a partially functional rumen at the end of the experiment.

\section{Supplementation with leucine and threonine affects energy metabolism in skeletal muscle}

The proteomic and metabolomic data obtained from skeletal muscle biopsies highlighted other two main metabolic adaptations. On one hand, the pathway analysis showed an increase in oxidative metabolism as components of NADH dehydrogenase (Complex I) (NADH and NDUFAB1) and cytochrome c oxidase (Complex IV) (COX2 and COX5A) [33] were increased 
in the LT group, indicating an increase in the activity of the mitochondrial respiratory chain and oxidative phosphorylation (OXPHOS). OXPHOS would be fueled by a higher rate of the citrate cycle, as suggested by an upregulation of enzymes (ACO2) and metabolites (2-oxoglutarate, GTP and NADH). The ADP/ATP carrier protein SLC25A4, involved in the exchange of cytoplasmic ADP with mitochondrial ATP across the mitochondrial inner membrane, was also found at higher abundance. It is interesting to note that these proteins $(\mathrm{COX} 2, \mathrm{COX} 5 \mathrm{~A}$ and NDUFAB1, as well as VCP) clustered positively in the correlation a. alysis in muscle (Figure 3A). The citrate cycle would be more active due to the fuel proicis by BCAA, however it has been shown that it could also be fueled by an increase in fa $v$ acid degradation as carnitine, involved in the transport of fatty acids to the mitochondr ${ }_{\iota}$ and HADHA, involved in fatty acid beta-oxidation, had both higher abundances in the $\mathrm{I}$ ', roup. Positive correlation between muscle leucine with carnitine in muscle and serur. ar $d$ with HADHA in muscle, may also be an indicative of this metabolic adaptation. 1.' metabolites acetoacetate and 3-hydroxybutyrate also indicated an increase in the metabo ${ }^{1:} \mathrm{sm} \sim$. Acetyl CoA. Nutrients (BCAA, butyrate and fats) are metabolized to acetyl-CoA, whi h suvsequently enters the citrate cycle to finally produce ATP as energy for cellular process^c $[21]$. Consequently, increased ATP availability leads to increased ATP transfer from mitoci ondria (upregulation of SLC25A4) as well as transport across membranes in other organelles (upregulation of the ATPase VCP).

On the other hand, the increased abundance of PRDX1 and SOD2 in the muscle tissue pointed towards the activation of superoxide radicals removal (GO:0019430) [35]. Since OXPHOS is not completely efficient and not every oxygen receives four electrons, a greater amount of superoxide anions and peroxides are likely produced under OXPHOS activation. The increase in antioxidant defenses may be a compensatory mechanism for the increase in oxygen radicals. 
Another potential effect of leucine is its ability to increase protein synthesis through its effect on the mTOR complex 1 (mTORC1) which is known for its central regulation of cell metabolism, growth, proliferation, and survival [36]. There is evidence that leucine [37] as well as its metabolite 2-oxoisocaproate are able to activate mTOR $[31,38]$. Herein, we use the phosphorylation of the ribosomal protein S6, downstream of the mTORC1 signaling pathway, as an indicator of the mTORC1 function, directly related to the upregulation of protein synthesis [39]. In our case, the ratio of phospho-S6/S6 indicated a significan $\iota_{\text {n. }}$ regulation of mTORC1 in the LT group. Our results suggest that the supplemented leucim s...ulated the mTORC1 pathway and protein synthesis, which may have contribute to the slight but significant increase in the protein percentage observed in LT calves after C $7-\therefore$ aging performed at the same time than biopsies (manuscript in preparation).

Our present hypothesis is that supplementatic ? with Leu-Thr would positively affect skeletal muscle growth (increase of muscular as s) jy affecting two mechanisms: on one side, improving ribosomal protein synt' - esis 'hrough the phosphorylation of S6 (putatively via mTOR) and, on the other side, by provicing enough energy (ATP) to drive protein synthesis, which is energy-consuming.

\section{Supplementation with leucine and threonine affects plasma composition}

The metabolic effects were also visualized in plasma, due to the appearance of two oxidation/citrate cycle components (carnitine and 2-oxoglutarate), ketone bodies (3hydroxybutyrate and acetoacetate) and, as mentioned above, BCAA derivatives (2oxoisocaproate and 3-hydroxyisobutyrate). 
Changes in the plasma proteome indicated that leucine/threonine supplementation may have complex effects in the defense mechanisms of the organism (oxidative stress and native immune system): haptoglobin was not altered (Table S4), CRP and PON1 increased, ITIH4 decreased. PON1 is involved in detoxification and binds to HDL, preventing and delaying the oxidation of HDL and low-density lipoprotein (LDL) and therefore inhibiting the onset and progression of oxidative stress [40], whereas CRP and ITIH4 are acute phase proteins upregulated in acute phase response [41], although CRP is considered a minor acute phas: protein in bovine [42]. On

the other hand, a decrease in several serpin 3A isoforms was r serpin superfamily of protease inhibitors and it is encoded $\iota:$ a cluster of eight closely related genes in cattle [43]. The functionality of serpins remains . clear, but they may be involved in blood coagulation, fibrinolysis, cell migration, anc ' 10 none transport [44,45]. A hint on the potential physiological roles of different $\mathrm{s}_{\mathrm{V}} \mathrm{pd}^{\mathrm{r}} \mathrm{s}$ may be inferred from the correlation analysis, since some serpins (A3.1, A3.7) positive:y correlated with PON1, CRP and APOC3, whereas other serpins (A3.5, A3.6 and A3.8, sh st red together with ITIH4 and APOA2. Curiously, these two groups of proteins correlate negatively one to the other. In any case, further research is needed to clarify whether thes, metabolically tensed staı $s$ exist in these animals.

\section{Conclusions}

Supplementation with leucine and threonine increased oxidative metabolism in muscle through an increase in citrate cycle, the mitochondrial respiratory chain and OXPHOS. The energy substrates were the AA, which were catabolized to ketoacids mainly in the muscle and may yield 
acetyl-CoA to enter the citrate cycle. Other substrates were short fatty acids and/or products of lipid mobilization, which were also converted into acetyl-CoA to be oxidized. The generation of oxidative stress in the mitochondria was balanced by the upregulation of antioxidant proteins. Lastly, a complex readjustment of the plasma defense systems also occurred.

In conclusion, an integrative approach by combining metabolomics, proteomics and clinical chemistry allowed to characterize metabolic adaptations occurring in these animals, even in the absence of macroscopic changes in productive parameters. More stu ties to analyze the role of the rumen, adipose tissue and liver, and the metabolomic stı $\mathrm{v}$ ol hydrophobic compounds would extend our understanding about the metabolism i $1 \mathrm{a}_{c}{ }^{i}$ ry calves and may help to optimize their nutritional management.

\section{Availability of data and mater'a's}

The datasets during and/or anal ' ed curing the current study are available from the corresponding authors on reas $n$ ` ble request.

\section{Abbreviations}

AA: amino acid(s); BCAA: branched-chain amino acid(s); BCKA: branched-chain keto acid(s); BW: body weight; CT: computed tomography; DM: dry matter; MR: milk replacer; mTOR: mammalian target of rapamycin; PON1: paraoxonase-1; TSP: total protein staining 


\section{Supplementary material}

Table S 1 Diet management of Ctrl and LT groups during the experiment.

Table S 2. Starter concentrate feeding program.

Table S 3. Performance and food intake of calves fed a milk replacer supplemented with $0.3 \%$ leucine plus $0.3 \%$ threonine (LT) or a non-supplemented milk replacer.

Table S 4. Concentration of serum biochemical analytes and AA profile.

Table S 5. Raw proteomic data of muscle samples.

Table S 6. Raw proteomic data of serum samples

Table S 7. PANTHER GO-Slim analysis of biological pro'e ' ${ }^{\prime}$ an'. molecular functions of the differential proteins between Ctrl and LT groups identified in skeletal $\mathrm{r}$ uscl .

Table S 8. PANTHER GO-Slim analysis of biolo: : al process and molecular functions of the differential proteins between Ctrl and LT groups identified in nlasn $^{\mathrm{l}}$.

Table S 9. Reactome Pathway Analy is irr the differential proteins and metabolites identified in skeletal muscle and serum between Ctrl and LT gren.

Fig. S 1. Principal component a talyses (PCA) of muscle (a) and serum (b) chemometrics data.

Fig. S2. Correlation plots of quantified metabolites in muscle versus serum. Dark blue and dark red colors indicate stronger positive and negative correlation, respectively. Pearson $r$ coefficients were calculated and significant level was set at $p<0.05$. The larger size of the circle represents a high correlation coefficient (approaching to 1 ) whereas smaller size of the circle indicate the opposite (approaching to 0 ). Non-significant correlations are depicted with a cross on top of the circle.

\section{Author Contributions}


K.Y. performed the experimental sessions in metabolomics and proteomics, data analysis, bioinformatics, statistics and manuscript writing. M.M. (manuscript, metabolomics and data analysis), A.H. (manuscript, proteomics and data analysis), M.T. (experimental design, manuscript, sampling, data analysis and statistics), A. Bach (experimental design, manuscript revision), J.K. (manuscript and proteomics), N.Y., L.A., E.R. and R.P (analytical work), N.G. (analytical work and data analysis), A.A. and P.D. E. (manuscript revision), A. Bassols (experimental design, analytical work supervision, manuscript writı. ${ }_{\text {` }}$ and revision). All authors read and approved the final manuscript.

\section{Acknowledgements}

We thank Dr. Charles Pineau for the invaluabı advice on the manuscript orientation, Ms. Bruna

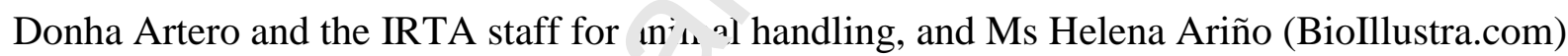
for the illustrations. This research , as supported by the Spanish Ministry of Economy and Competitivity (AGL2015-6846, C2-1-P to MT and AGL2015-68463-C2-2-P to AB). The author KY acknowledges Chi na Sch', larship Council (CSC) for the graduate scholarship program's funding.

\section{Conflict of Interest}

The authors declare no conflict of interest.

\section{References}


[1] P. Li, Y.-L. Yin, D. Li, S. Woo Kim, G. Wu, Amino acids and immune function, Br. J. Nutr. 98 (2007) 237-252. doi:10.1017/S000711450769936X.

[2] G. Wu, Amino acids: Metabolism, functions, and nutrition, Amino Acids. 37 (2009) 1-17. doi:10.1007/s00726-009-0269-0.

[3] J. Escobar, J.W. Frank, A. Suryawan, H. V. Nguyen, S.R. Kimball, L.S. Jefferson, T.A. Davis, Physiological rise in plasma leucine stimulates musc! _ protein synthesis in neonatal pigs by enhancing translation initiation factor activation $\leftarrow m$ J. Physiol. Metab. 288 (2005) E914-E921. doi:10.1152/ajpendo.00510.20C.

[4] Y. Yin, K. Yao, Z. Liu, M. Gong, Z. Ruan, D. Ceng, 3. Tan, Z. Liu, G. Wu, Supplementing 1-leucine to a low-proteir liet increases tissue protein synthesis in weanling pigs, Amino Acids. 39 (20ıㄴ 1477-1486. doi:10.1007/s00726-010-0612-5.

[5] J.A.B. Pedroso, T.T. Zampieri, J L unato, Reviewing the effects of l-leucine supplementation in the re ula ${ }^{\text {tion }}$ of food intake, energy balance, and glucose homeostasis, Nutrient 7 ( 2015) 3914-3937. doi:10.3390/nu7053914.

[6] Y. Duan, F. Li, Y. . i, Y. Tang, X. Kong, Z. Feng, T.G. Anthony, M. Watford, Y. Hou, G. $\mathrm{Wu}, \mathrm{Y}$. Yin, The role of leucine and its metabolites in protein and energy metabolism, Amino Acids. 48 (2016) 41-51. doi:10.1007/s00726-015-2067-1.

[7] A. Bonvini, A.Y. Coqueiro, J. Tirapegui, P.C. Calder, M.M. Rogero, Immunomodulatory role of branched-chain amino acids, Nutr. Rev. 76 (2018) 840-856. doi:10.1093/nutrit/nuy037. 
[8] J. Kanjanapruthipong, Supplementation of milk replacers containing soy protein with threonine, methionine, and lysine in the diets of calves., J. Dairy Sci. 81 (1998) 29122915. doi:10.3168/jds.S0022-0302(98)75852-7.

[9] D. Li, C. Xiao, S. Qiao, J. Zhang, J.E. W., P.A. Thacker, Effects of dietary threonine on performance, plasma parameters and immune function of growing pigs, Anim. Feed Sci. Technol. 78 (1999) 179-188. doi:10.1016/S0377-8401(99)ก0005-X.

[10] M. Herrero, C. Simõ, V. García-Cañas, E. Ibáñez, A. Cifuı nte s, Foodomics: MS-based strategies in modern food science and nutrition, Ma Sputrom. Rev. 31 (2012) 49-69. doi:10.1002/mas.20335.

[11] K. Yu, M. Matzapetakis, D. Valent, Y. S ‘ $`$, r.M. De Almeida, M. Terré, A. Bassols, Skeletal muscle metabolomics and bl dd biochemistry analysis reveal metabolic changes associated with dietary amino ar: 1 s. pplementation in dairy calves., Sci. Rep. 8 (2018) 13850. doi:10.1038/s41598.01え $32241-4$.

[12] K. Yu, F. Canalias, D. Sol ^-Oriol, L. Arroyo, R. Pato, Y. Saco, M. Terré, A. Bassols, Age-

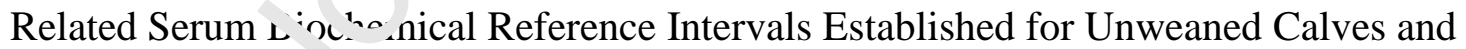
Piglets in the Post-weaning Period, Front. Vet. Sci. 6 (2019) 123. doi:10.3389/fvets.2019.00123.

[13] J.R. Wiśniewski, A. Zougman, N. Nagaraj, M. Mann, Universal sample preparation method for proteome analysis., Nat. Methods. 6 (2009) 359-62. doi:10.1038/nmeth.1322.

[14] A. Horvatić, N. Guillemin, H. Kaab, D. McKeegan, E. O’Reilly, M. Bain, J. Kuleš, P.D. Eckersall, Quantitative proteomics using tandem mass tags in relation to the acute phase 
protein response in chicken challenged with Escherichia coli lipopolysaccharide endotoxin, J. Proteomics. 192 (2019) 64-77. doi:10.1016/j.jprot.2018.08.009.

[15] S. Martinez-Subiela, A. Horvatic, D. Escribano, L. Pardo-Marin, M. Kocaturk, V. Mrljak, R. Burchmore, J.J. Ceron, Z. Yilmaz, Identification of novel biomarkers for treatment monitoring in canine leishmaniosis by high-resolution quantitative proteomic analysis, Vet. Immunol. Immunopathol. 191 (2017) 60-67. doi:10.1016/j.vetimm.2017.08.004.

[16] L. Gaita, B. Manzi, R. Sacco, C. Lintas, L. Altieri, F. Lnm:ar i, T.L. Pawlowski, M. Redman, D.W. Craig, M.J. Huentelman, S. Ober-Rc:noıus, S. Brautigam, R. Melmed, C.J. Smith, J. Marsillach, J. Camps, P. Curatolo, \& M.. Persico, Decreased serum arylesterase activity in autism spectrum dis r'eles, Psychiatry Res. 180 (2010) 105-113. doi:10.1016/J.PSYCHRES.2010.04 J10.

[17] G.A.N. Gowda, Y.N. Gowda, F. Rai ${ }^{+}$ery, Massive Glutamine Cyclization to Pyroglutamic Acid in Human Serum Disc Jvt:•d Using NMR Spectroscopy, Anal. Chem. 87 (2015) 3800-3805. doi:10.102^'ač-94435b.

[18] D. Jacob, C. Deı rri M. Lefebvre, M. Maucourt, A. Moing, NMRProcFlow: a graphical and interactive tool dedicated to 1D spectra processing for NMR-based metabolomics, Metabolomics. 13 (2017) 36. doi:10.1007/s11306-017-1178-y.

[19] H. Wickham, R. François, L. Henry, K. Müller, dplyr: A Grammar of Data Manipulation, (2019).

[20] H. Wickham, Package reshape2, (2017). 
[21] A. Kassambara, ggpubr: "ggplot2" Based Publication Ready Plots, (n.d.).

[22] H. Wickham, ggplot2: Elegant Graphics for Data Analysis, Second Edi, Springer, 2016. doi:10.18637/jss.v035.b01.

[23] W. Taiyun, S. Viliam, L. Michael, X. Yihui, J. Yan, Z. Jeff, corrplot: Visualization of a Correlation Matrix, (2017).

[24] J. Gross, U. Ligges, Tests for Normality [R package norter.

[25] A. Walker, openxlsx: Read, Write and Edit XLSX Files, (2019).

[26] A. Fabregat, S. Jupe, L. Matthews, K. Sidiropou'os, M. Gillespie, P. Garapati, R. Haw, B. Jassal, F. Korninger, B. May, M. Milacic. C..' Roca, K. Rothfels, C. Sevilla, V. Shamovsky, S. Shorser, T. Varusai, _ Viteri, J. Weiser, G. Wu, L. Stein, H. Hermjakob, P. D’Eustachio, The Reactome Patı vay Knowledgebase, Nucleic Acids Res. 46 (2018) D649-D655. doi:10.1093/n ‘/ $\varepsilon^{k_{\lambda}+132 .}$

[27] Y. Perez-Riverol, A. Fsor 'as, J. Bai, M. Bernal-Llinares, S. Hewapathirana, D.J. Kundu, A. Inuganti, J. Cris G. Mayer, M. Eisenacher, E. Pérez, J. Uszkoreit, J. Pfeuffer, T. Sachsenberg, Ş. Iımaz, S. Tiwary, J. Cox, E. Audain, M. Walzer, A.F. Jarnuczak, T. Ternent, A. Brazma, J.A. Vizcaíno, The PRIDE database and related tools and resources in 2019: improving support for quantification data, Nucleic Acids Res. 47 (2019) D442D450. doi:10.1093/nar/gky1106.

[28] M. Holeček, Branched-chain amino acids in health and disease: Metabolism, alterations in blood plasma, and as supplements, Nutr. Metab. 15 (2018) 33. doi:10.1186/s12986-018- 
0271-1.

[29] A.E. Harper, R.H. Miller, K.P. Block, Branched-chain amino acid metabolism, 1984.

[30] C.B. Newgard, Interplay between lipids and branched-chain amino acids in development of insulin resistance., Cell Metab. 15 (2012) 606-14. doi:10.1016/j.cmet.2012.01.024.

[31] D.A. Columbus, M.L. Fiorotto, T.A. Davis, Leucine is a major regulator of muscle protein synthesis in neonates, Amino Acids. 47 (2015) 259-270. $\therefore$. 1 1 (.1007/s00726-014-1866-0.

[32] M. Aviram, M. Rosenblat, C.L. Bisgaier, R.S. Newtın, I L. Primo-parmo, B.N. La Du, Paraoxonase Inhibits High-density Lipoprotein C vidr cion and Preserves its Functions, J. Clin. Invest. 101 (1998) 1581-1590.

[33] D.C. Wallace, Mitochondrial disease. 'n man and mouse, Science (80-. ). 283 (1999) 1482-1488. doi:10.1126/science 2১? 5407.1482.

[34] M.G. Vander Heiden, L.C. ``antley, C.B. Thompson, Understanding the warburg effect: The metabolic requiremen s of cell proliferation, Science (80-. ). 324 (2009) 1029-1033. doi:10.1126/sci-nc: 1150809 .

[35] Y. Collins, E.T. Chouchani, A.M. James, K.E. Menger, H.M. Cochemé, M.P. Murphy, Mitochondrial redox signalling at a glance., J. Cell Sci. 125 (2012) 801-6. doi:10.1242/jcs.098475.

[36] M. Laplante, D.M. Sabatini, mTOR signaling at a glance., J. Cell Sci. 122 (2009) 358994. doi:10.1242/jcs.051011.

[37] P.J. Atherton, K. Smith, T. Etheridge, D. Rankin, M.J. Rennie, Distinct anabolic signalling 
responses to amino acids in C2C12 skeletal muscle cells, Amino Acids. 38 (2010) 15331539. doi:10.1007/s00726-009-0377-x.

[38] J. Escobar, J.W. Frank, A. Suryawan, H. V Nguyen, C.G. Van Horn, S.M. Hutson, T.A. Davis, Leucine and $\alpha$-Ketoisocaproic Acid, but Not Norleucine, Stimulate Skeletal Muscle Protein Synthesis in Neonatal Pigs, J. Nutr. 140 (2010) 1418-1424. doi:10.3945/jn.110.123042.

[39] X.M. Ma, J. Blenis, Molecular mechanisms of mTOR-mec “atr d translational control, Nat. Rev. Mol. Cell Biol. 10 (2009) 307-318. doi:10.102"'/nrın 2672.

[40] M. Antoncic-Svetina, R. Turk, A. Svetina, D. G.res, 3. Rekic, D. Juretic, Lipid status, paraoxonase-1 activity and metabolic nar me $\mathrm{rrs}$ in serum of heifers and lactating cows related to oxidative stress, Res. Vet. s 1. 90 (2011) 298-300.

doi:10.1016/j.rvsc.2010.05.022

[41] M. Neumaier, G. Metak, IM.t. Scherer, C-reactive protein as a parameter of surgical trauma: CRP responsı aft $\epsilon_{\iota}$ different types of surgery in 349 hip fractures, Acta Orthop. 77 (2006) 788-ı). ^r : $: 10.1080 / 17453670610013006$.

[42] F. Ceciliani, J.J. Ceron, P.D. Eckersall, H. Sauerwein, Acute phase proteins in ruminants, J. Proteomics. (2012). doi:10.1016/j.jprot.2012.04.004.

[43] A. Péré-Brissaud, X. Blanchet, D. Delourme, P. Pélissier, L. Forestier, A. Delavaud, N. Duprat, B. Picard, A. Maftah, L. Brémaud, Expression of SERPINA3s in cattle: focus on bovSERPINA3-7 reveals specific involvement in skeletal muscle, Open Biol. 5 (2015) 150071. doi:10.1098/rsob.150071. 
[44] P.G.W. Gettins, Serpin structure, mechanism, and function., Chem. Rev. 102 (2002)

4751-804. http://www.ncbi.nlm.nih.gov/pubmed/12475206 (accessed July 18, 2019).

[45] G.A. Silverman, P.I. Bird, R.W. Carrell, F.C. Church, P.B. Coughlin, P.G.W. Gettins, J.A. Irving, D.A. Lomas, C.J. Luke, R.W. Moyer, P.A. Pemberton, E. Remold-O’Donnell, G.S. Salvesen, J. Travis, J.C. Whisstock, The Serpins Are an Expanding Superfamily of Structurally Similar but Functionally Diverse Proteins, J. Biol. Chem. 276 (2001) 3329333296. doi:10.1074/jbc.R100016200.

\section{Figures}

Figure 1. a. Western blot of Phospho-S6 a d $S 5$ wih their corresponding total protein staining in each animal; b. Boxplots of S6, Phosph S6 and the ratio of Phospho-S6/S6 with comparison $p$ values between Ctrl and LT. Mean inte is i ies were normalized and scaled to 1.

Figure 2. Principal comp nen analyses (PCA) with loadings of quantified metabolites from muscle (a) and serum (k)

Figure 3. Correlation plots of quantified metabolites in muscle (a) and serum (b). Dark blue and dark red colors indicate stronger positive and negative correlation, respectively. Pearson $r$ coefficients were calculated and significant level was set at $p<0.05$. The larger size of the circle represents a high correlation coefficient (approaching to 1) whereas smaller size of the circle 
indicate the opposite (approaching to 0 ). Non-significant correlations are depicted with a cross on top of the circle. Compounds are grouped by hierarchical clustering order.

Figure 4. Schematic representation of the putative metabolic effects exerted by leucine/threonine supplementation in the MR in pre-weaned calves. 


\section{Tables}

Table 1. Concentration of serum analytes and plasma AA which were found significantly different between Ctrl and LT groups (see Table S 4 for complete results).

\begin{tabular}{|c|c|c|c|}
\hline Analyte & Ctrl & $\mathbf{L T}$ & $p$-value \\
\hline \multicolumn{4}{|l|}{ Serum Clinical Chemistry } \\
\hline Cholesterol (mg/dL) & $60.29 \pm 4.8$ & $9: 5 u-9.48$ & 0.041 \\
\hline Glutathione Peroxidase (GPx) (U/L) & $423.84 \pm 38.22$ & $321.89 \pm 16.71$ & 0.031 \\
\hline Triglyceride (mg/dL) & $19.69 \pm 2.2 t$ & $40.26 \pm 11.21$ & 0.058 \\
\hline \multicolumn{4}{|l|}{ Plasma AA $(\mu \mathrm{M})$} \\
\hline Histidine & $71.45 \pm 4.53$ & $92.29 \pm 9.06$ & 0.048 \\
\hline Isoleucine & $94.72 \pm 3.52$ & $127.28 \pm 13.37$ & 0.017 \\
\hline Leucine & $164.74 \pm 10.36$ & $220.37 \pm 28.84$ & 0.056 \\
\hline
\end{tabular}


Table 2. Proteins with significantly differential abundances in skeletal muscle identified in Ctrl and LT groups.

\begin{tabular}{|c|c|c|c|}
\hline $\begin{array}{l}\text { Gene name } \\
\text { (Bos taurus) }\end{array}$ & Protein description & $p$-value & $\begin{array}{l}\text { fold change } \\
\text { (LT vs Ctrl) }\end{array}$ \\
\hline HADHA & Trifunctional enzyme subunit alpha, mitochondrial & 0.037 & 0.37 \\
\hline PRDX1 & Peroxiredoxin-1 & 0.030 & 0.26 \\
\hline SOD2 & Superoxide dismutase 2 , mitochondrial & 0.043 & 0.26 \\
\hline SLC25A4 & ADP/ATP-carrier protein & 0.041 & 0.21 \\
\hline VCP & Transitional endoplasmic reticulur 1 \& $\mathrm{CP}\lrcorner \mathrm{se}$ & 0.041 & 0.20 \\
\hline $\mathrm{COX} 2$ & Cytochrome c oxidase & 0.030 & 0.19 \\
\hline COX5A & Cytochrome $\mathrm{c}$ oxidase, $s \mathrm{sb}$ nit $5 \mathrm{~A}$, mitochondrial & 0.030 & 0.19 \\
\hline NDUFAB1 & Chain U, Acyl Ca -ier Protein, mitochondrial & 0.041 & 0.19 \\
\hline $\mathrm{ACO} 2$ & Acor:tas 2, $\mathrm{r}$ itochondrial & 0.071 & 0.11 \\
\hline GYS1 & Glycogen synthase 1 protein & 0.025 & -0.29 \\
\hline RTN2 & Reticulon-2 & 0.010 & -0.25 \\
\hline
\end{tabular}


Table 3. Proteins with significantly differential abundances in plasma identified in Ctrl and LT groups.

\begin{tabular}{|c|c|c|c|}
\hline $\begin{array}{l}\text { Gene name } \\
\text { (Bos taurus) }\end{array}$ & Protein description & $p$-value & $\begin{array}{l}\text { fold change } \\
\text { (LT vs Ctrl) }\end{array}$ \\
\hline PON1 & Paraoxonase 1 & 0.004 & 0.41 \\
\hline APOA2 & apolipoprotein A-II & 0.679 & 0.35 \\
\hline CRP & C-reactive protein & 0.025 & 0.35 \\
\hline APOC3 & Apolipoprotein C-III & 0.015 & 0.33 \\
\hline $\mathrm{F} 2$ & Prothrombin & 0.041 & 0.20 \\
\hline SERPINA3-5 & Serpin A3-5 & 0.018 & -0.44 \\
\hline SERPINA3-6 & Serpin A3-6 & 0.018 & -0.44 \\
\hline ITIH4 & Inter-alpha-trvp $: \eta$ inhibitor heavy chain $\mathrm{H} 4$ & 0.015 & -0.34 \\
\hline SERPINA3-8 & Serrin $13-8$ & 0.006 & -0.31 \\
\hline FN1 & Fibronectin & 0.034 & -0.24 \\
\hline SERPINA3-7 & Serpin A3-7 & 0.041 & -0.20 \\
\hline SERPINA3-2 & Serpin A3-2 & 0.013 & -0.19 \\
\hline SERPINA3-1 & Serpin A3-1 & 0.018 & -0.19 \\
\hline
\end{tabular}


Table 4. Metabolites (mean $\pm \mathrm{SE}, \mu \mathrm{Mol} / 100 \mathrm{~g}$ muscle) with significantly differential concentration in skeletal muscle identified in Ctrl and LT groups.

\begin{tabular}{|c|c|c|c|}
\hline Metabolite & Ctrl & $\mathbf{L T}$ & $p$-value \\
\hline 2-Oxoglutarate & $0.80 \pm 0.07$ & $1.22 \pm 0.10$ & 0.005 \\
\hline Acetate & $1.08 \pm 0.12$ & $1.45 \pm 0.13$ & 0.052 \\
\hline Acetoacetate & $0.52 \pm 0.04$ & $0.67 \pm \iota 05$ & 0.035 \\
\hline GTP & $0.50 \pm 0.03$ & $0 . \iota^{7} \pm 0.02$ & 0.005 \\
\hline Leucine & $0.51 \pm 0.0$ & $0 . / 0 \pm 0.07$ & 0.042 \\
\hline NADH & $r .21=0 . \iota 4$ & $0.35 \pm 0.05$ & 0.049 \\
\hline Valine & $94 \pm 0.06$ & $1.30 \pm 0.11$ & 0.013 \\
\hline
\end{tabular}


Table 5. Metabolites (mean $\pm \mathrm{SE}, \mu \mathrm{M}$ ) with significantly differential concentration in serum identified in Ctrl and LT groups.

\begin{tabular}{|c|c|c|c|}
\hline Metabolite & Ctrl & LT & $p$-value \\
\hline 2-Oxobutyrate & $6.93 \pm 0.71$ & $10.00 \pm 1.15$ & 0.042 \\
\hline 2-Oxoisocaproate & $4.09 \pm 0.26$ & $5.66 \pm 0.39$ & 0.002 \\
\hline 3-Hydroxybutyrate & $44.73 \pm 4.11$ & $71.01 \pm$ ‘ 57 & 0.021 \\
\hline 3-Hydroxyisobutyrate & $9.34 \pm 0.79$ & $110 ; \pm 0.57$ & 0.034 \\
\hline Asparagine & $25.24 \pm 1 .<\angle$ & $30.17 \pm 1.37$ & 0.028 \\
\hline Creatinine & 1. $86 \simeq 1.40$ & $19.57 \pm 1.15$ & 0.064 \\
\hline Formate & $35.59 \pm 2.37$ & $30.60 \pm 1.46$ & 0.08 \\
\hline Fructose & $119.11 \pm 10.24$ & $83.50 \pm 11.51$ & 0.039 \\
\hline Histidine & $10.87 \pm 1.21$ & $16.70 \pm 1.88$ & 0.023 \\
\hline Isoleucinc & $39.70 \pm 2.24$ & $50.31 \pm 3.32$ & 0.021 \\
\hline Leucine & $64.06 \pm 3.96$ & $81.19 \pm 6.02$ & 0.035 \\
\hline Proline & $53.79 \pm 1.60$ & $64.51 \pm 2.40$ & 0.002 \\
\hline Serine & $51.36 \pm 2.40$ & $68.80 \pm 4.81$ & 0.007 \\
\hline Threonine & $66.43 \pm 5.00$ & $78.94 \pm 3.60$ & 0.065 \\
\hline
\end{tabular}


Valine

Carnitine

Citrulline

Methylsuccinate
$59.11 \pm 3.58$

$5.76 \pm 0.25$

$8.10 \pm 0.48$

0.001

$39.16 \pm 3.10$

$49.77 \pm 1.39$

0.009

$4.46 \pm 0.40$

$5.51 \pm 0.32$

0.061 


\section{Journal Pre-proof}

Table 6. Joint pathway analyses of upregulated proteins and metabolites in muscle and blood using MetaboAnalyst.

\begin{tabular}{|c|c|c|c|c|c|c|c|c|c|c|c|c|c|c|c|c|}
\hline \multirow[t]{2}{*}{ Pathway } & \multicolumn{8}{|c|}{ Muscle (9 proteins and 9 metabolites) } & \multicolumn{8}{|c|}{ Blood (5 proteins and 16 metabolites) } \\
\hline & Protein & Metabolite & hit & $\begin{array}{c}p- \\
\text { value }\end{array}$ & $-\log (p)$ & $\begin{array}{l}\text { Holm } \\
p\end{array}$ & FDR & Impact & Protein & Metabolite & hit & $\begin{array}{c}p- \\
\text { value }\end{array}$ & $-\log (p)$ & $\begin{array}{l}\text { Holm } \\
p\end{array}$ & FDR & Impact \\
\hline $\begin{array}{l}\text { Valine, } \\
\text { leucine } \\
\text { and } \\
\text { isoleucine } \\
\text { degradatio } \\
\mathrm{n}\end{array}$ & HADHA & $\begin{array}{c}\text { leucine, } \\
\text { acetoacetate, } \\
\text { valine, } \\
\text { isoleucine }\end{array}$ & $5 / 82$ & $\begin{array}{c}6.69 \mathrm{E} \\
-05\end{array}$ & 9.6 & 0.005 & 0 & 0.18 & - & $\begin{array}{l}\text { leucine, } \\
\text { isoleucine, } \\
\text { vali ıe, } \\
\text { ox ss cc nrr ate, } \\
\text { - -hyc :oxy } \\
\text { isobutyrate }\end{array}$ & $5 / 82$ & $\begin{array}{l}5.66 \mathrm{E} \\
-04\end{array}$ & 7.5 & 0.04 & 0.02 & 0.13 \\
\hline $\begin{array}{l}\text { Butanoate } \\
\text { metabolis } \\
\mathrm{m}\end{array}$ & HADHA & $\begin{array}{c}2- \\
\text { oxoglutarate, } \\
\text { acetoacetate }\end{array}$ & $3 / 47$ & 0.003 & 5.9 & 0.21 & 0.07 & 0.34 & & - & - & & & - & - & \\
\hline $\begin{array}{l}\text { Aminoacy } \\
\text { 1-tRNA } \\
\text { metabolis } \\
\mathrm{m}\end{array}$ & - & $\begin{array}{c}\text { leucine, } \\
\text { isoleucine, } \\
\text { valine }\end{array}$ & $3 / 87$ & 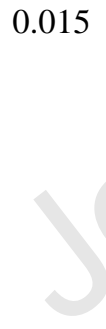 & 4.2 & & 0.2 & 0.04 & - & $\begin{array}{c}\text { leucine, } \\
\text { isoleucine, } \\
\text { alanine, } \\
\text { valine, } \\
\text { serine, } \\
\text { proline, } \\
\text { histidine, } \\
\text { asgaragine }\end{array}$ & $8 / 87$ & $\begin{array}{l}1.74 \mathrm{E} \\
-07\end{array}$ & 15.6 & $\begin{array}{l}1.39 \mathrm{E}- \\
05\end{array}$ & $\begin{array}{l}1.39 \\
\text { E-05 }\end{array}$ & 0.13 \\
\hline $\begin{array}{l}\text { Citrate } \\
\text { cycle }\end{array}$ & $\mathrm{ACO} 2$ & $\begin{array}{c}2- \\
\text { oxoglutarate }\end{array}$ & $2 / 50$ & 0.039 & 3.2 & 1 & 0.62 & 0.3 & - & - & - & & & - & - & \\
\hline
\end{tabular}

$p$-value, $p$-values of significant pathways in enrichment analysis; Impact, impact of the pathway according to the pathway topology analysis. Higher -log( $p)$

means more significant pathway; higher impact means a higher percentage of hit compounds 


\section{Significance}

Leucine and threonine are essential amino acids for the pre-weaning calf, being of high importance for growth. In this study, we found that leucine and threonine supplementation of milk replacer to feed pre-weaning calves led to differences in the proteome, metabolome and clinical chemistry analytes in skeletal muscle and plasma, albeit no differences in productive performance were recorded. This study extends our understanding on the metabolism in dairy calves and helps optimizing their nutritional status. 


\section{Conflict of Interest}

The authors declare no conflict of interest. 


\section{Highlights}

- Leucine and threonine are essential amino acids for calves

- Calves received milk replacer supplemented or not with $3 \%$ leucine and $3 \%$ threonine

- Supplementation with Thr and Leu leads to higher citrate cycle activity and OXPHOS

- Supplementation with Leu and Thr increased antioxidant del nses

- Supplementation with Leu and Thr increases the mus, le F 1ospho-S6/S6 ratio 
a

Phospho-S6
v)
ज小
रा (4)
siv
sit
(स) समt
s.

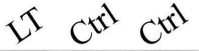
st $4(x)$

TPS

S6

TPS

b

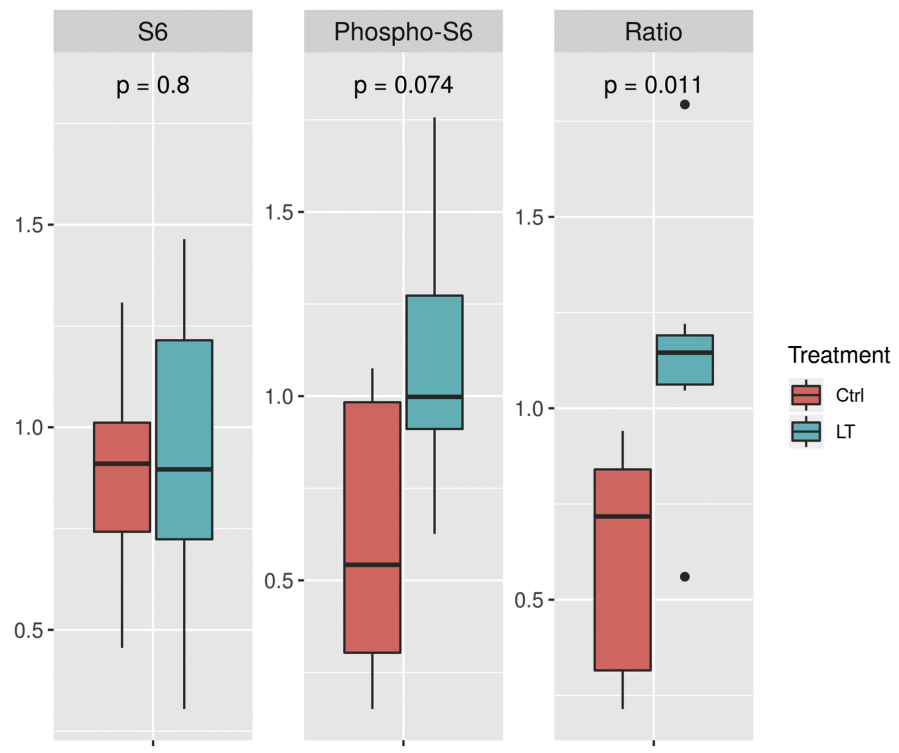

Figure 1 


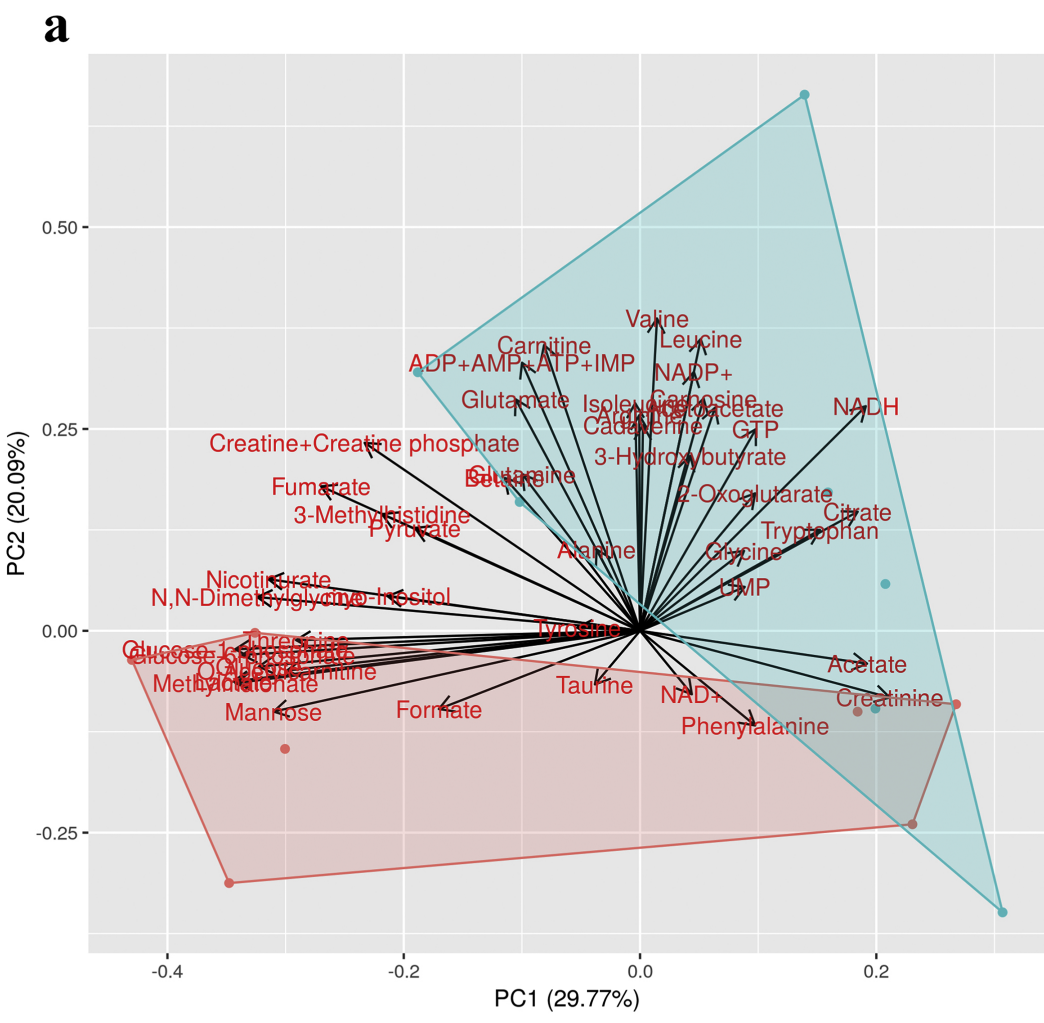

Treatment

\begin{tabular}{|l|l}
\hline- & Ctrl \\
\hline \hline- & LT
\end{tabular}

b

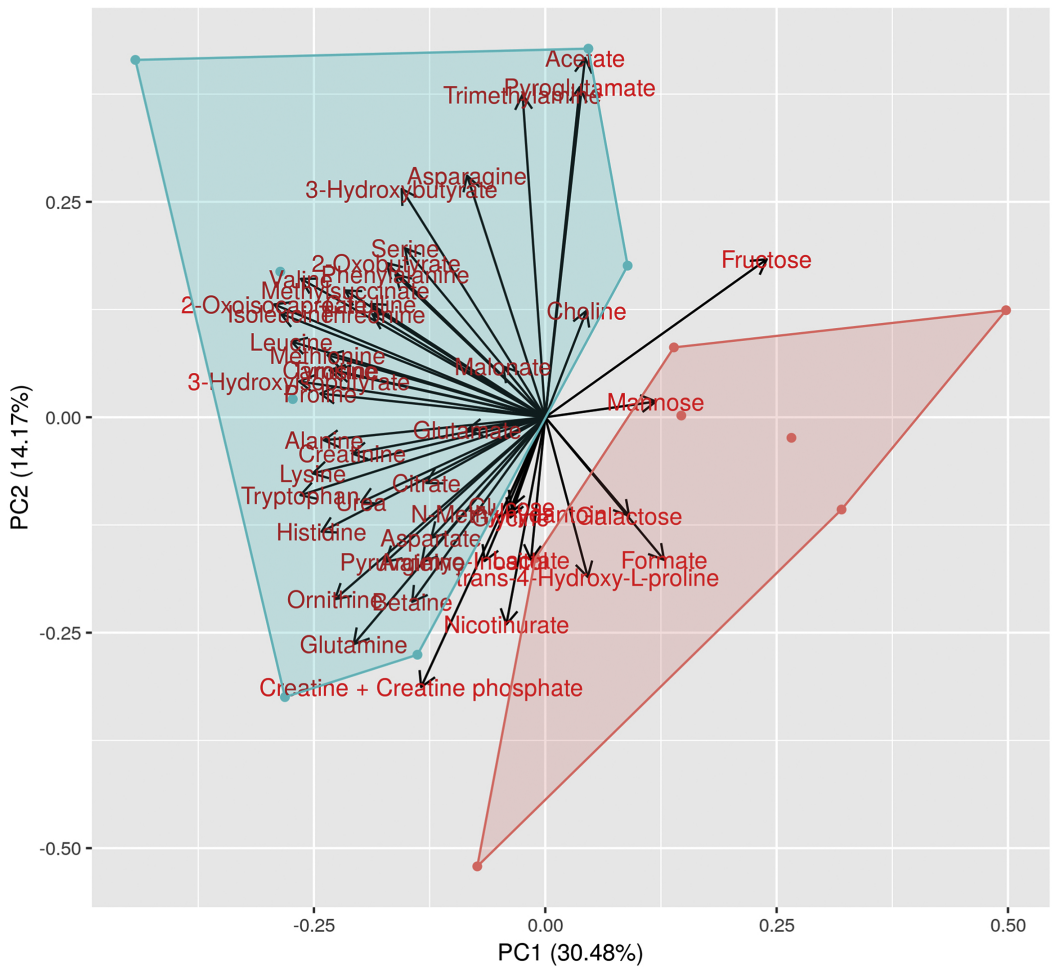

Treatment

\begin{tabular}{|l|l}
\hline- & Ctrl \\
\hline \hline- & LT
\end{tabular}

Figure 2 
6.

Pyruvate $X 0 . X X X X X X X X X X X X X X X X X X X X X X X O X X X X \quad X X X X X X X X X X X X X X X X X X X X X$

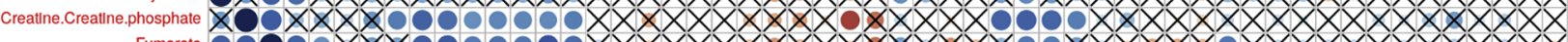
Fumarate $0000 \times X X 0000000 \times X X X X X X X X X X X X X X X X 00 \times X X X X X 0 X X X X X X X X X X X X X$ Threonne OX0000000000000XXXXXXXOXXXXXXXXXXXXXXXXXXXXXXXXXXXXXXX хз.Methylistilline XX0000XX00X0X00XXXXXXXOXXXXXXXXXXXXXXXXXXXXXXXXXXXXXXX

myo.Inositol XXX0000000000000XXXXXXXOXXOXXXXXXXXXXXXXXXXXXXXXXXXXXXX

Mannose XXXOXO0000000000XXXXXXOXXOXXXXXXXXXXXXXXXXXXXXXXXXOXXX

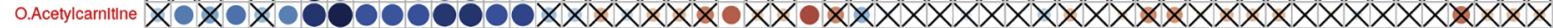
N.N.DImethylglyclne X00000000000000 XXXXXXXXXX0XXXXXXXXXXXXXXXXXXXXXXXXXXXXXXX

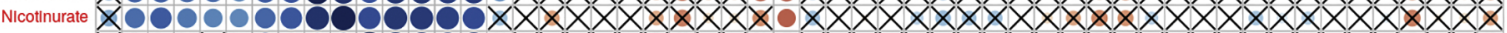

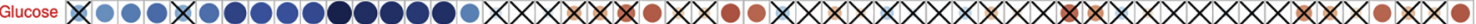

Lactate X00000000000000XXXXXX0XX00XXXXXXXXXXX0XXXXXXXXXXX0XXX

Methylmalonate X000X0000000000XXXXXXOXXOOXXXXXXXXXXXXXXXXXXXXXXXOXXX Glucose.1.phosphate X000000000000000XXXXXOOXXXOXXXXXXXXXXXXXXXXXXXXXXXXXXX

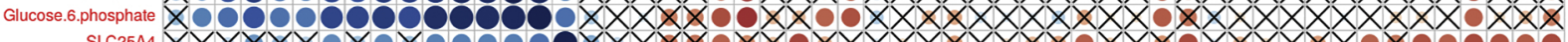

SLC25A4XXXXXXOXOX000000XXXXXOXXOXXXXXXXXXX0XXX0XXXXXX0X000000

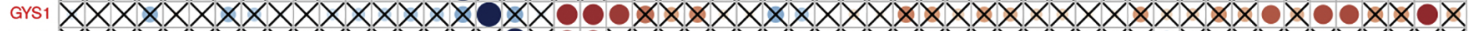

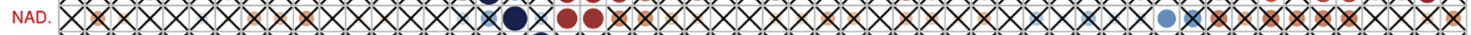

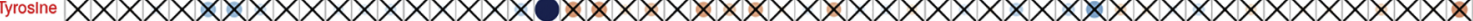

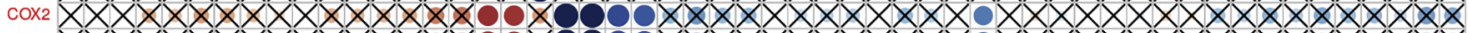
$\cos _{5 \mathrm{~A}} \times \times \times \times \times \times \times \times \times \times \times \times \times \times \times \times 00 \times 000 \times \times \times \times \times \times \times \times \times \times \times \times 0 \times \times \times \times \times \times \times \times \times \times \times \times \times \times \times \times \times \times$

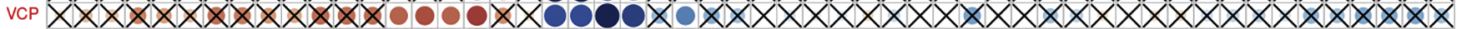

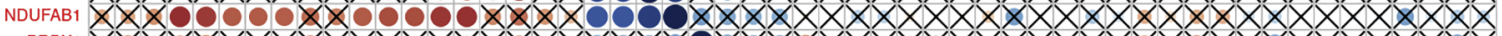

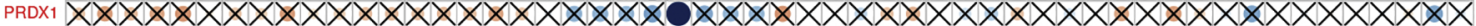

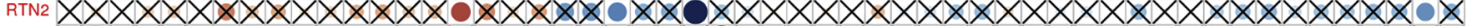

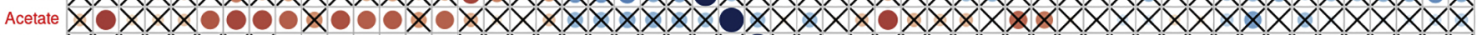

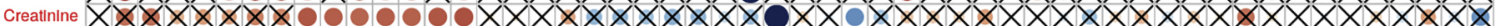

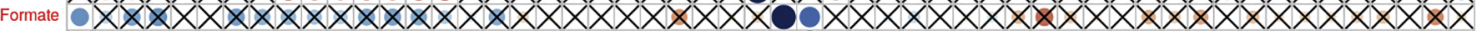

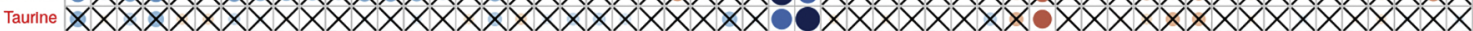

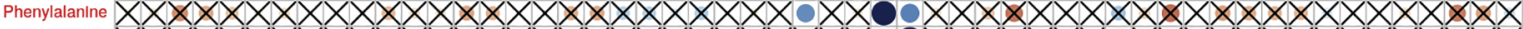

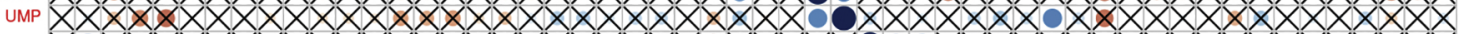

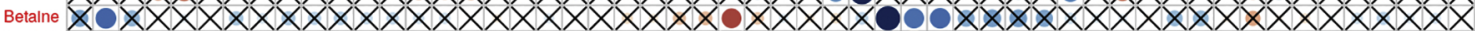

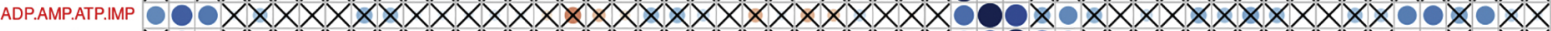

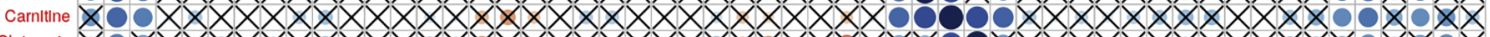

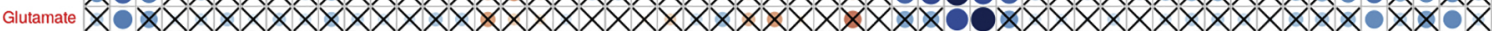

$\mathrm{AcO}_{2} \times \times \times \times \times \times \times \times \times \times \times \times \times \times \times 0 \times \times \times 00 \times \times \times \times \times \times \times \times \times \times \times 00 \times 0 \times \times \times \times \times \times \times \times \times \times \times \times \times \times \times \times 0 \times$

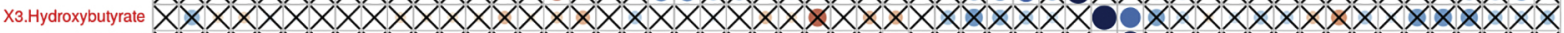

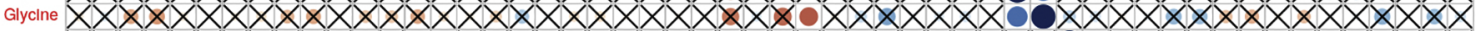

Girate XXXXXXXXXXXOXOOXXXXXXXXXXXXXXXOXXXXXXXOOXXXXXXXXXXXXXX

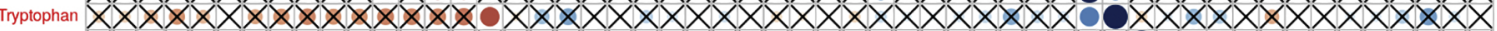

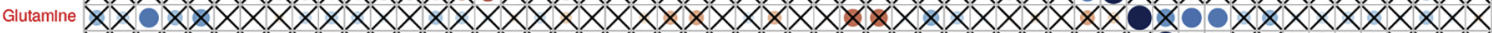

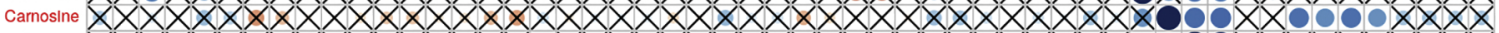

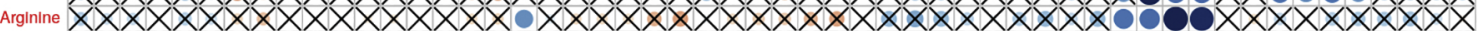
cadaverne XXXXXXXXXXXXXXXXXXXXXXXXXXXXXXXXXXXXXXXX0000XXXXXXXXXX

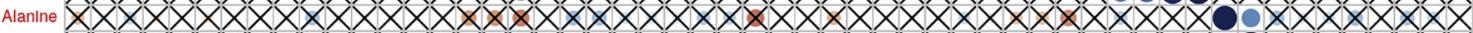

son2 $\times \times \times \times \times \times \times \times \times \times \times \times \times \times \times \times \times \times \times \times \times \times \times \times \times \times \times \times \times \times \times \times \times \times \times \times \times \times \times \times \times \times \times \times 00 \times \times \times \times \times \times \times \times$

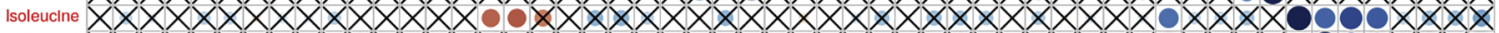

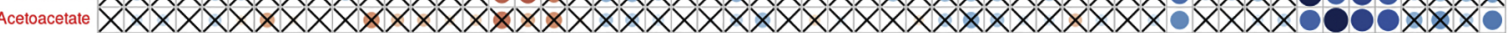
Leucine XXXXXXXXXXXXXXX00XXXXXXXXXXXXXXX00XXXXXXXOXXXX000000X0

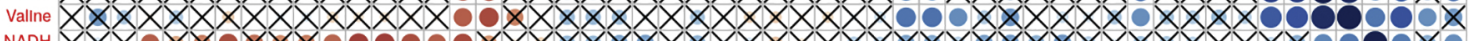

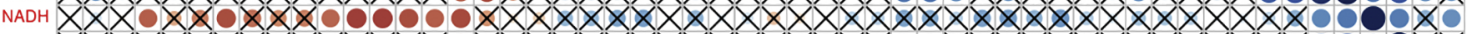

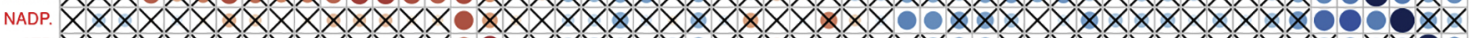

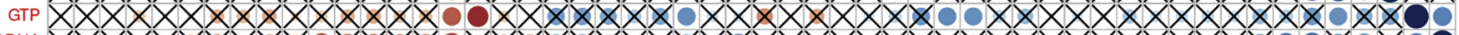

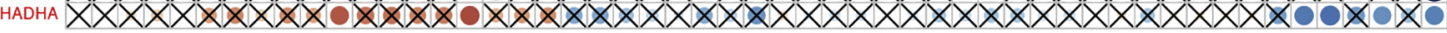




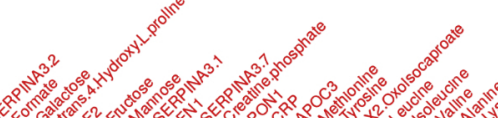

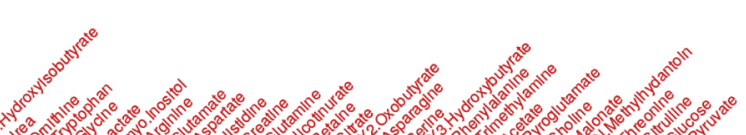

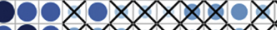

$10 \times 000$

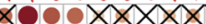

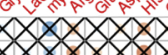

Galactose

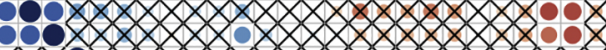

Fructose $\times 1.80$

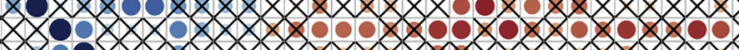

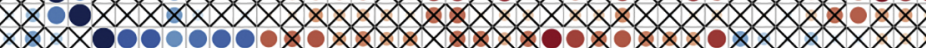

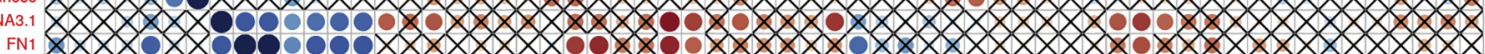

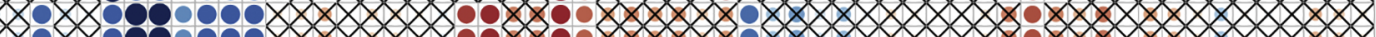

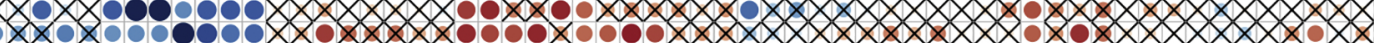

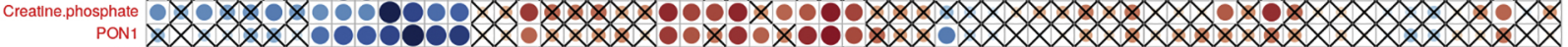

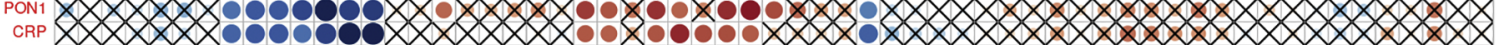

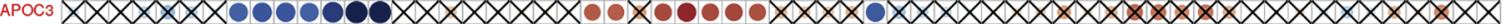

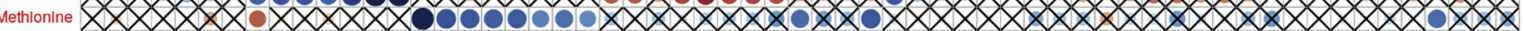

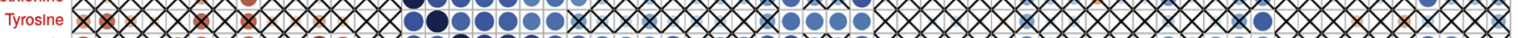

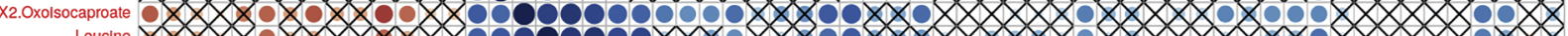

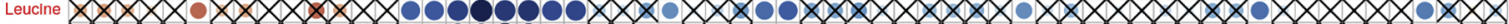

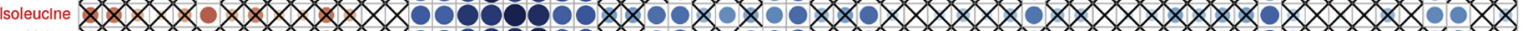

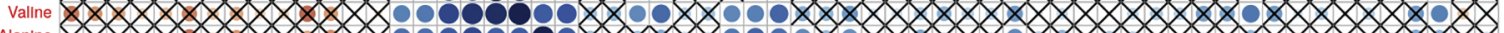

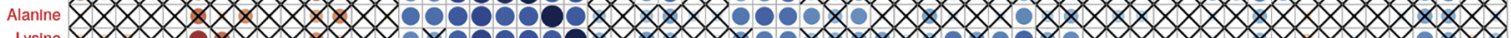

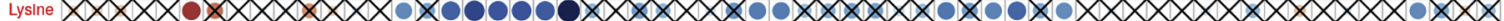

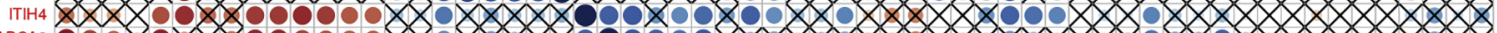

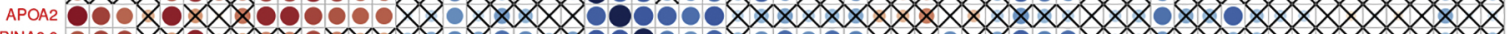

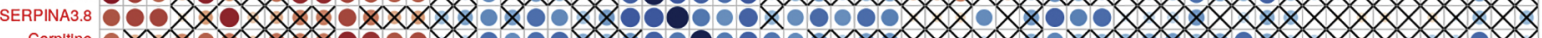

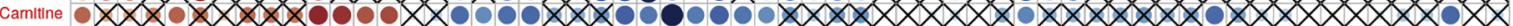

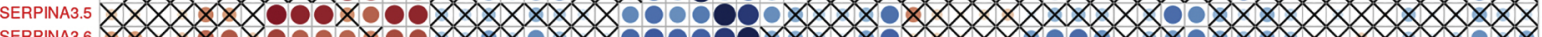

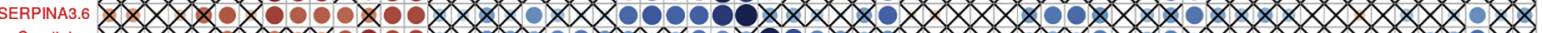

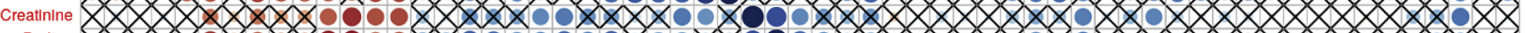

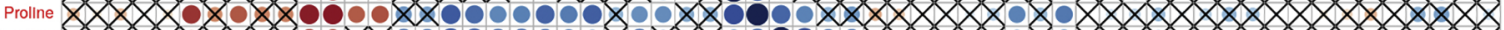

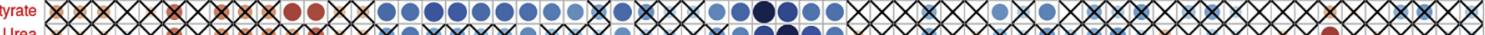

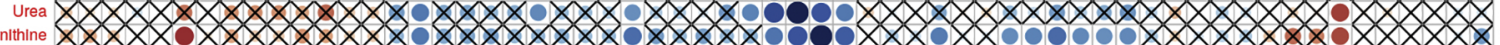

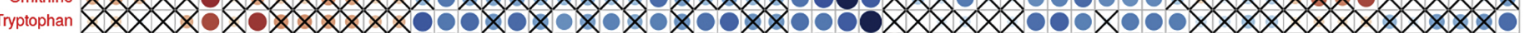

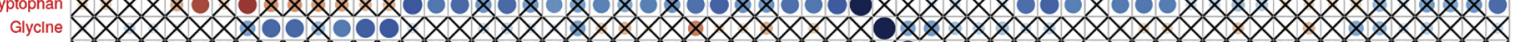

myo.Inositol 7 La

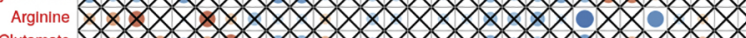

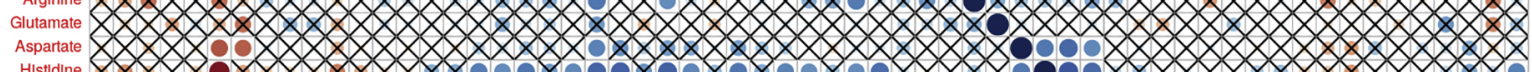

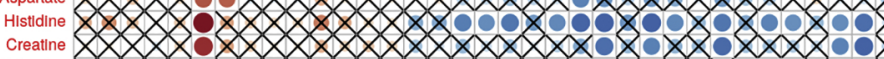

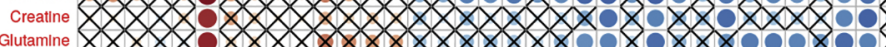

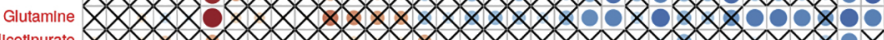

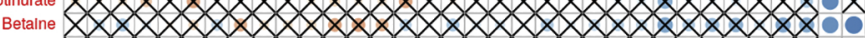

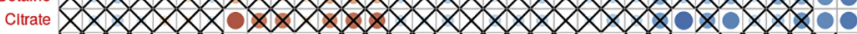

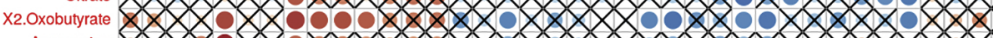

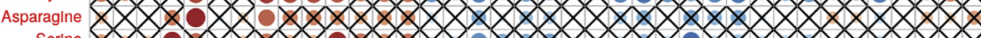

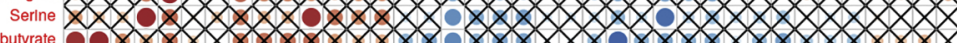

Phenylalanine 0

Pyroglutamate

Pyroglutamate

Choline $1 \times 2 \times$ W

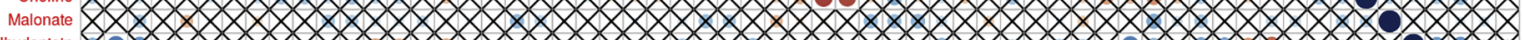

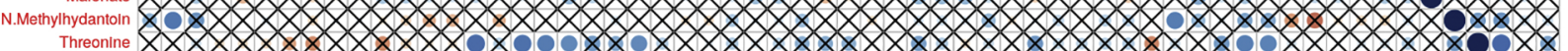

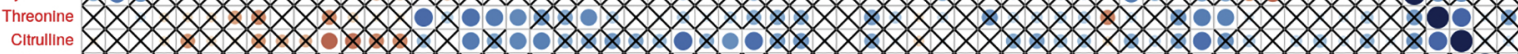

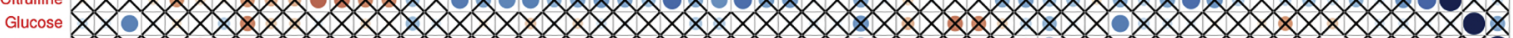

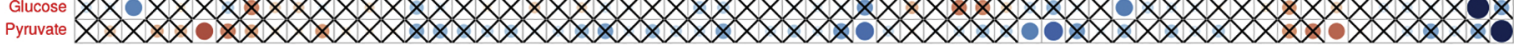




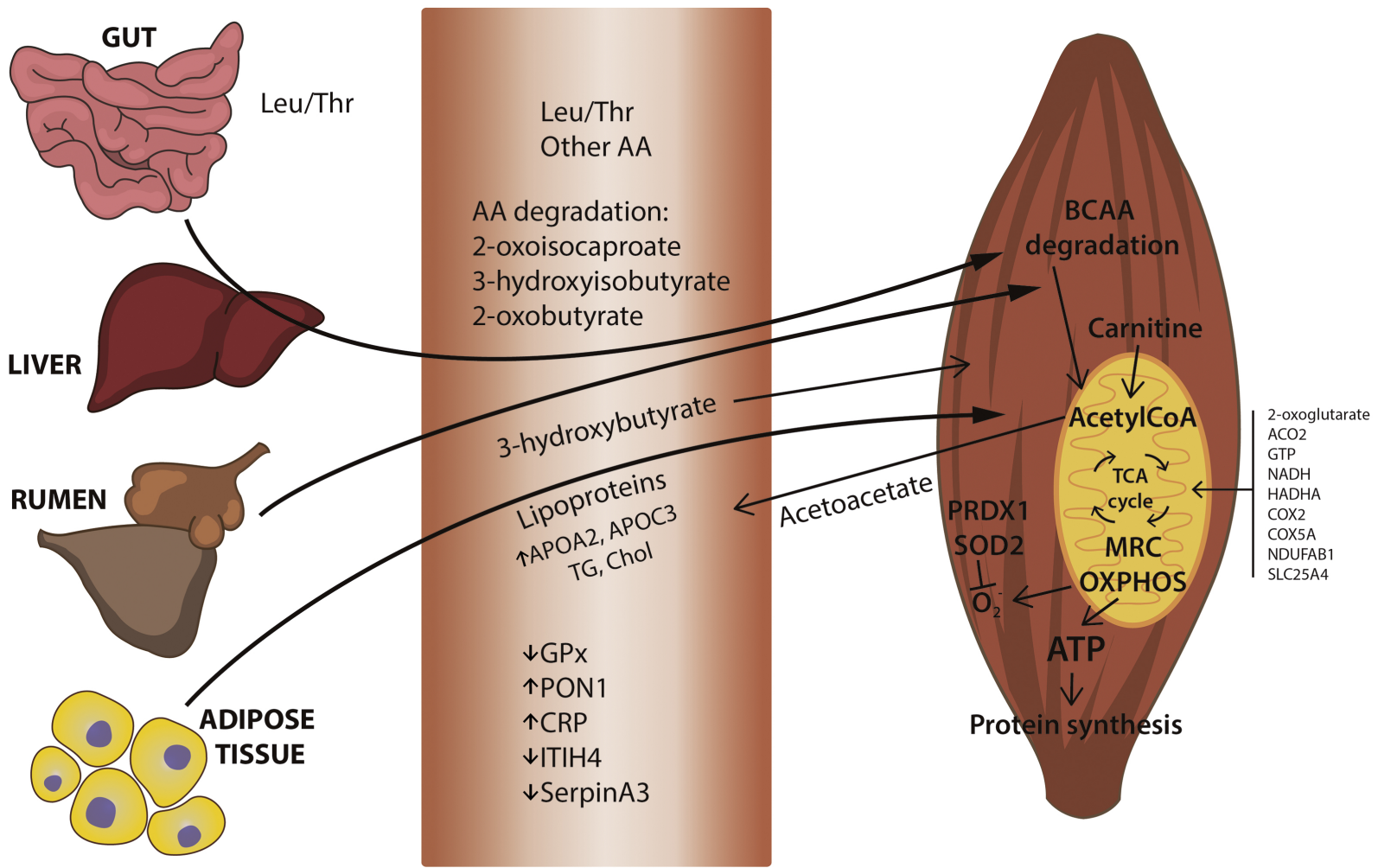

PLASMA

SKELETAL MUSCLE

Figure 4 


\section{$\mathbf{a}$}

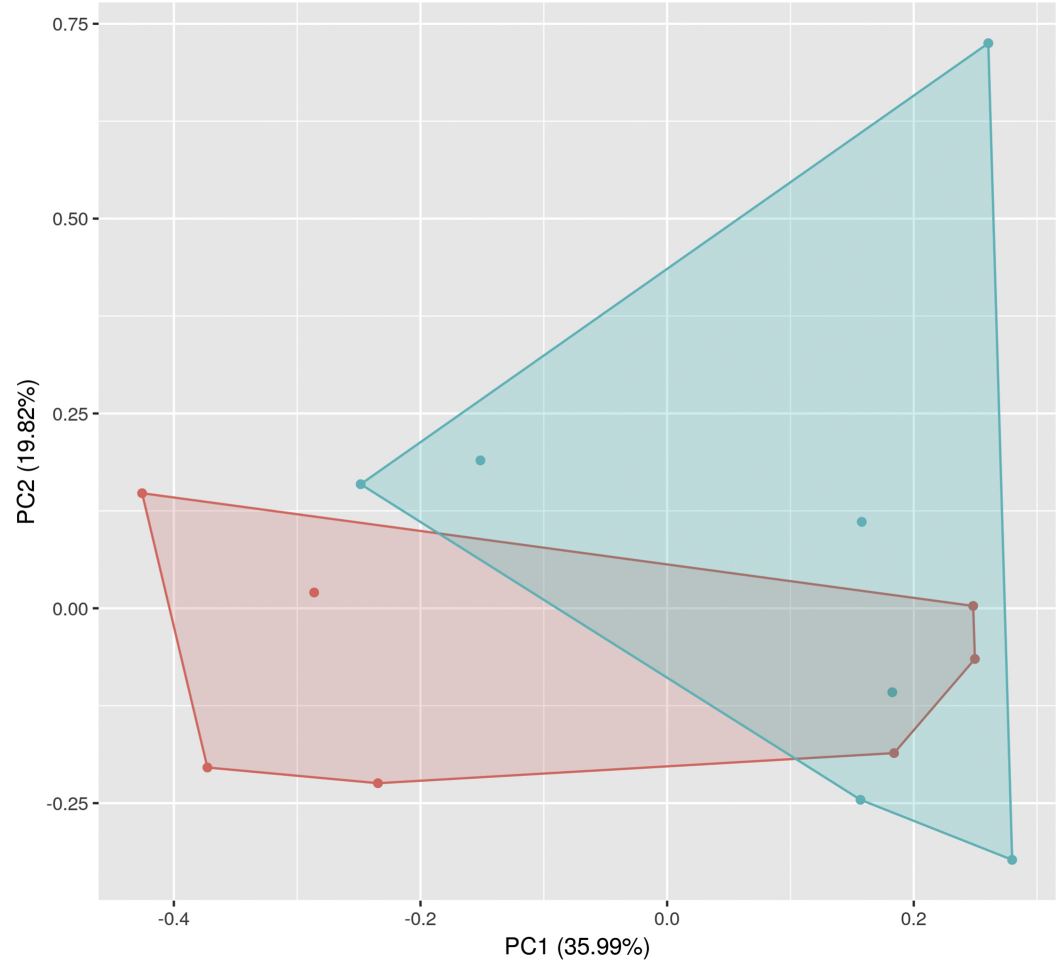

Treatment

\begin{tabular}{|l|l}
\hline- & Ctrl \\
\hline- & LT
\end{tabular}

b

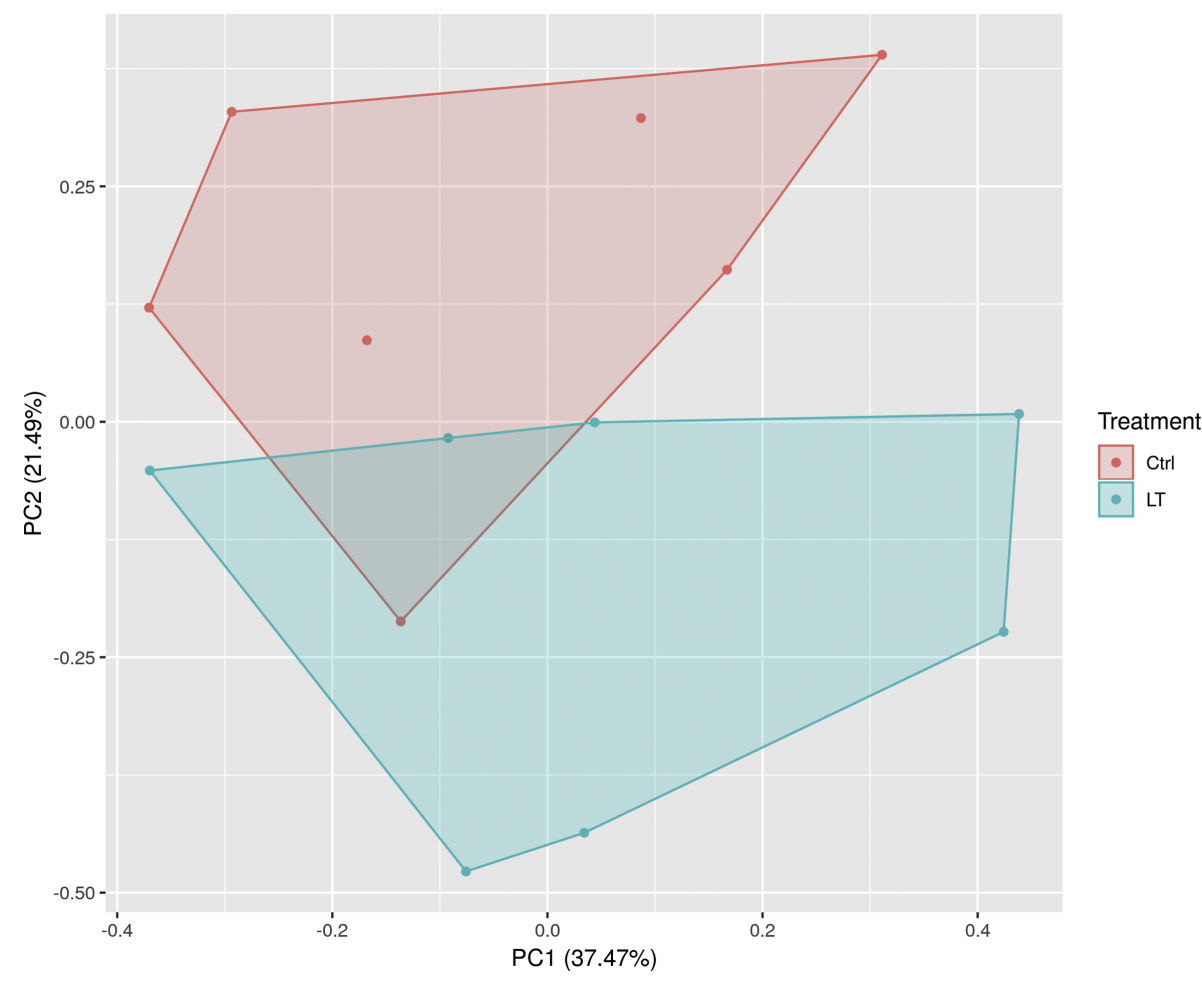

Figure 5 
Macte Aograse

Mads Nodoss

Macle ADPANP RTRM

Macie Narea

Made Avirat

Macle Evare

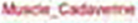

Made Campra

Mude Cromon

Macle Crais

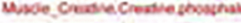

Macie Coustrma

Miscie forma

Masa Aurara

Maste Cloon

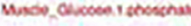

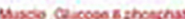

Mucie Qustanat

Mucie Casarm

Mocie, Shon

Uascle ar

Mabe- buevela

Minde, Lactail

Mnele Levor

Miscie Monnete

Mace Metrymatonas

Uncos frophoses

Mesce NN Dimetroforor

Made Nap.

Macie NUDit

unce nop

Macle, Nootruta

Wasta OAserpeannasa

Mode Pervilateve

Hacie Pryas

uncie, Tuestre

Mucie Drences a

weacle tristapn

Mode Frour

Macte un

Wucie_Vatine

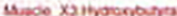

Made xometrinatidie

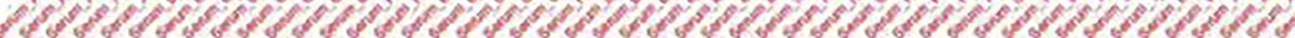

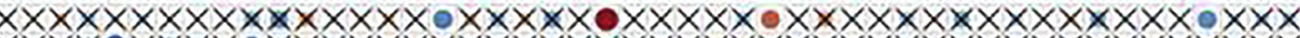
$\times \times \times \times 0 \times \times \times \times 0 \times \times \times \times \times \times \times \times \times \times \times \times \times \times \times \times \times \times \times \times \times \times \times \times \times \times \times \times \times \times \times \times \times \times \times \times \times$ $\times \times \times \times \times \times 0 \times \times \times \times \times \times \times \times \times \times \times \times \times \times \times \times \times \times \times \times \times \times \times \times \times \times \times \times \times \times \times \times \times \times \times \times \times \times \times \times \times$

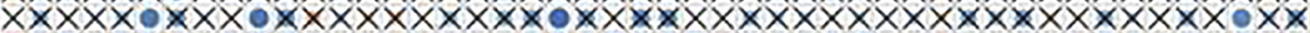

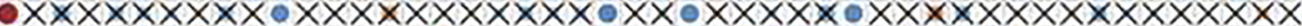
$\times \times \times \times \times \times \times \times \times \times \times \times \times \times \times \times \times \times \times \times \times \times 0 \times \times \times \times \times \times \times 0 \times \times \times \times \times \times \times \times \times \times \times \times \times \times \times \times \times$

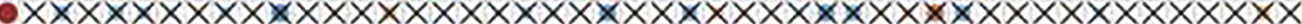
$\times \times \times \times \times \times 0 \times \times \times \times 0 \times \times \times 0 \times \times \times \times \times \times \times \times \times \times \times \times \times \times 0 \times \times \times \times \times \times \times \times \times \times \times \times \times \times \times \times \times$

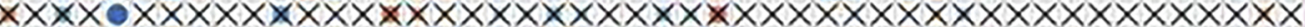

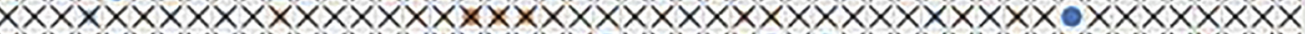
$\times \times \times \times \times \times \times \times \times \times \times \times \times \times \times \times \times \times \times \times 0 \times \times \times \times \times 0 \times 0 \times \times \times \times \times \times \times \times \times \times \times \times \times \times \times \times \times$ $\times 0 \times \times \times \times \times \times \times \times \times \times \times \times \times \times \times \times \times 0 \times \times \times \times \times \times \times \times 0 \times \times \times \times \times \times \times \times \times \times \times \times \times \times \times \times \times \times \times$ $\times \times \times 0 \times \times \times \times \times \times \times \times \times 0 \times \times \times \times \times \times \times \times \times \times \times \times \times \times \times \times \times \times \times \times \times \times \times \times 0 \times \times \times \times \times \times \times \times \times$ - $\times \times \times \times \times \times \times \times \times \times \times \times \times \times \times \times \times 0 \times \times \times \times \times \times \times \times \times \times \times 0 \times \times \times 0 \times \times \times \times 0 \times \times \times \times \times \times \times \times$

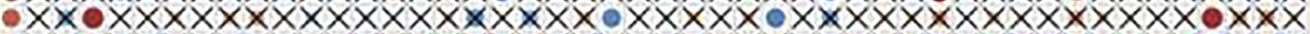

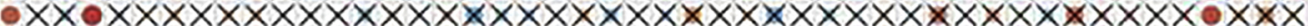
$\times \times \times 0 \times \times \times \times \times \times \times \times \times \times \times \times \times 0 \times \times \times \times \times \times \times \times \times \times 0 \times \times \times \times \times \times \times \times \times \times \times \times \times \times \times 0 \times \times \times$ $\times \times \times \times \times \times 0 \times \times \times \times 0 \times \times 0 \times \times \times \times \times \times \times \times \times \times \times 0 \times \times \times 0 \times \times \times \times \times \times \times \times \times \times \times \times \times \times \times \times \times$

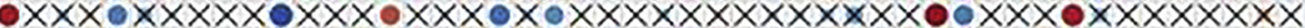
$\times \times \times \times \times \times \times \times \times \times \times \times \times \times \times \times \times \times \times \times \times \times \times \times \times \times \times \times \times \times \times \times \times \times \times \times \times \times \times \times \times \times \times \times \times \times$

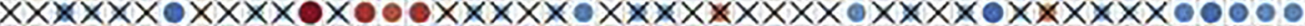
$\times \times \times \times 0 \times \times \times \times 0 \times \times \times \times \times \times \times \times \times \times \times \times \times \times 0 \times \times \times \times \times \times \times \times \times \times \times \times \times \times \times \times \times \times \times \times \times \times \times$ $\times \times \times 0 \times \times \times \times \times \times \times \times \times \times \times \times \times \times \times \times \times \times \times \times \times \times \times \times 0 \times \times \times \times \times \times \times \times \times \times \times \times \times \times \times 0 \times \times \times$

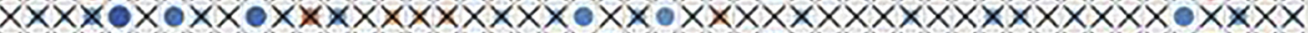

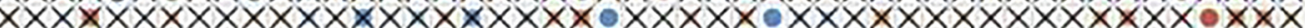

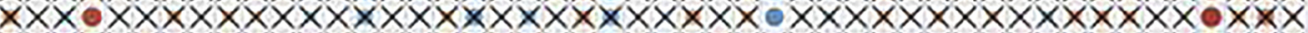

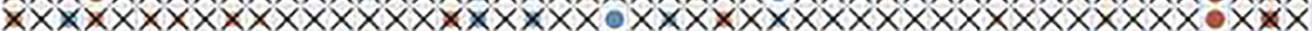
$\times \times \times \times \times \times \times \times \times \times \times \times \times \times \times \times \times \times \times \times \times \times \times \times \times \times \times \times 0 \times \times \times \times \times \times \times \times \times \times \times \times \times \times \times 0 \times 0 \times$

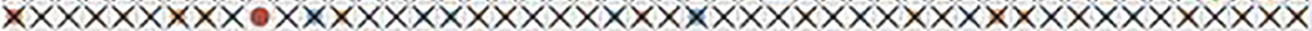
$\times \times \times \times \times \times 0 \times \times \times \times \times \times \times \times \times \times \times \times \times \times \times \times \times \times \times \times \times \times \times \times \times \times \times \times \times \times \times \times \times \times \times \times \times \times \times \times \times$

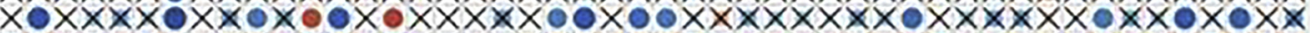
$\times \times \times \times \times \times \times \times \times \times \times \times \times \times \times \times \times \times \times \times \times \times \times \times \times \times \times \times \times \times \times \times \times \times \times \times \times \times \times \times \times \times \times 0 \times \times \times$ $\times \times \times \times \times \times \times \times \times \times \times \times \times \times \times \times \times \times \times \times \times \times \times \times \times \times \times \times \times \times \times \times \times \times \times \times \times \times \times \times \times \times \times 0 \times \times \times$ $\times \times \times \times \times \times \times \times \times \times 0 \times \times \times 0 \times \times \times \times \times 0 \times \times \times \times \times \times \times \times \times \times 0 \times \times 00 \times \times \times \times 0 \times \times \times \times \times \times \times 1$

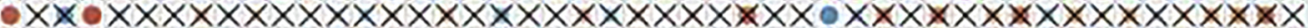
$\times \times \times \times \times \times \times \times \times \times \times \times 0 \times \times \times \times \times \times \times \times \times \times \times \times \times \times \times \times \times \times \times \times \times \times \times \times \times \times \times \times \times \times \times \times \times \times$

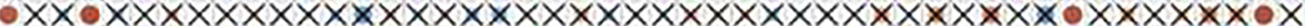

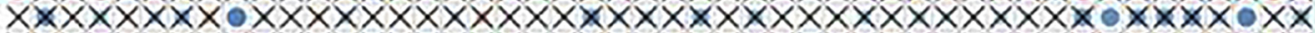
$\times \times \times \times \times \times \times \times \times \times \times \times \times \times \times \times \times \times \times \times \times \times \times \times \times \times \times \times \times \times \times \times \times \times \times \times \times \times \times \times \times \times \times \times \times \times \times \times$ $\times \times \times \times \times \times \times \times \times \times \times \times \times \times \times \times \times \times \times \times \times \times \times \times \times \times \times \times \times \times \times \times \times \times \times 0 \times \times \times 0 \times \times \times \times \times \times \times \times$

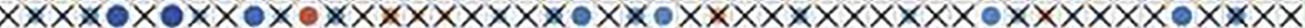
$\times \times \times \times \times \times \times \times \times \times \times \times \times \times \times \times \times \times \times \times \times \times \times \times \times \times \times \times \times \times \times \times \times \times \times \times \times \times \times \times \times \times \times \times \times \times \times \times$ 0 $\times \times \times 0 \times \times \times \times \times \times \times \times \times \times \times \times 0 \times \times \times \times \times \times \times \times 0 \times \times \times \times \times \times \times \times \times \times \times \times \times \times \times \times \times \times \times \times \times$ 